JOURNAL OF THE BRAZILIAN SOCIETY OF MECHANICAL SCIENCES AND ENGINEERING

(Springer Verlag, Impact Factor $=0.963)$

ISSN: 1678-5878 (print version)

ISSN: 1806-3691 (electronic version)

Accepted July $24^{\text {th }} 2016$

\title{
COMPUTATIONAL MODELLING OF MAGNETOHYDRODYNAMIC CONVECTION FROM A ROTATING CONE IN ORTHOTROPIC DARCIAN POROUS MEDIA
}

\author{
O. Anwar Bég* \\ Fluid Mechanics and Propulsion, Aeronautical \& Mechanical Engineering Department, School of \\ Computing, Science and Engineering, Newton Building, University of Salford, Manchester, M54WT, UK. \\ V. R. Prasad and B. Vasu \\ Dept. Mathematics, Madanapalle Institute of Technology and Science, Madanapalle-517325, India \\ R. S. R. Gorla \\ Heat Transfer, Dept. Mechanical Engineering, Cleveland State University, Cleveland, Ohio, USA. \\ * Author to whom correspondence should be addressed; Email-gortoab@gmail.com; $\underline{\text { O.A.Beg@salford.ac.uk }}$
}

\begin{abstract}
Free convective magnetohydrodynamic flow from a spinning vertical cone to an orthotropic Darcian porous medium under a transverse magnetic field is studied. The non-dimensionalized two-point boundary value problem is solved numerically using the Keller-Box implicit finite difference method. The effects of spin parameter, orthotropic permeability functions, Prandtl number and hydromagnetic number on flow characteristics are presented graphically. Tangential velocity and swirl velocity are accentuated with increasing permeability owing to a corresponding decrease in porous media resistance. Temperatures are depressed with increasing permeability. Validation of the solutions is achieved with earlier studies. Applications of the study arise in electromagnetic spin coating materials processing.
\end{abstract}

Key words: Rotating flow; Boundary Layers; Magnetohydrodynamics (MHD); Orthotropic Porous Medium; Darcy Number; Prandtl number; Swirl; Keller-Box Scheme; Magnetic Materials Processing.

\section{NOTATION}

\section{Dimensional}

$X$

$Y$

$\theta$

$R$

$(R)^{\prime}$

$r$

$U$

V co-ordinate parallel to cone surface

co-ordinate normal to cone surface

angular co-ordinate

radial co-ordinate

actual radius of cone

local radius of the cone

velocity component in $X$ direction

velocity component in $Y$ direction 


$\begin{array}{ll}W & \text { velocity component in } \\ T & \text { fluid temperature } \\ T_{w} & \text { cone surface temperature } \\ T_{\infty} & \text { free stream temperature } \\ U^{*} & \text { reference velocity } \\ B & \text { magnetic field strength } \\ g & \text { gravitational acceleration } \\ v & \text { kinematic viscosity of fluid } \\ \sigma & \text { electrical conductivity of fluid } \\ \rho & \text { density of fluid } \\ K_{X} & \text { permeability in } X \text { direction } \\ K_{\theta} & \text { permeability in } \theta \text { direction } \\ \alpha & \text { thermal diffusivity of the fluid } \\ \beta & \text { coefficient of thermal expansion of the fluid } \\ \Omega & \text { rotational velocity of the cone (spin velocity about symmetry axis) } \\ \phi & \text { semi-vertex angle of cone } \\ & \text { second order permeability tensor }\end{array}$

\section{Dimensionless}

$\begin{array}{ll}F & \text { similarity boundary layer stream function } \\ G & \text { similarity boundary layer rotational (swirl) velocity } \\ H & \text { similarity boundary layer temperature function } \\ x & \text { transformed X coordinate } \\ y & \text { transformed } Y \text { coordinate } \\ r & \text { transformed local cone radius } \\ u & \text { transformed X velocity } \\ v & \text { transformed } Y \text { velocity } \\ w & \text { transformed } \theta \text { velocity } \\ \kappa_{x} & \text { permeability function in } x \text {-direction (x-direction Darcy number) } \\ \kappa_{\theta} & \text { permeability function in } \theta \text {-direction ( } \theta \text {-direction Darcy number) } \\ P r & \text { Prandtl number } \\ \Phi & \text { non-dimensional temperature function } \\ G r_{L} & \text { local Grashof number }\end{array}$


Re rotational Reynolds number

Nmagnetohydrodynamic body force number

L reference scale length

\section{INTRODUCTION}

Exterior rotational hydrodynamic and thermal convection flows from bodies possessing axisymmetric geometries has been studied for a number of decades by engineers and mathematicians. The Coriolis forces experienced with rotation generated by the centrifugal field cause fluid to be impelled along the cone surface and substantially enhance heat transfer rates. Early work on rotating cone boundary layers was communicated by We [1]. Hering and Grosh [2] extended this analysis to incorporate heat transfer. Paterson et al. [3] considered the non-Newtonian flow and species transfer to a rotating cone. The convective heat transfer to both rotating cones and disks in powerlaw pseudoplastic fluids was reported by Smith and Grief [4]. Jeng and Chao [5] studied transpiration effects in transient convection on spinning disks and cones. Himasekhar and Sarma [6] obtained similarity solutions for the convection from a rotating cone in a stably-stratified medium. An excellent study of Newtonian laminar boundary layer convection on revolving disks and cones was presented by Lin and Lin [7]. A seminal study of mixed convection heat transfer in a non-Newtonian fluid from rotating cones/disks was reported by Wang and Kleinstreuer [8]. These theoretical investigations were largely directed at improving thermal designs of process equipment in the chemical industry. Gorla and Nakamura [9] analyzed the micropolar free and forced convective boundary layer flow from a spinning cone using a finite difference method. Experimental studies of laminar rotating convection have also been reported. Mao et al. [10] used a holographic technique to achieve improved visualization of the heat transfer regime on a rotating cone. More recently Chen et al. [11] investigated the dynamics of liquid film formation and evaporation on the inner surface of a rotating cone with different cone angles. Venkateswarlu et al. [12] also studied the mass transfer of ionic solutions on a rotating cone electrode. Anilkumar and Roy [13] discussed self-similar analytical solutions for the transient free and forced convection from a rotating cone configuration. Ozturk and Ece [14] studied the transient convection from a spinning body. They showed that fluid near the surface of rotation is forced radially outwards with a simultaneous upward flow induced tangential to the spinning body surface. This tangential flow boosts 
convection and elevates the rate of heat transfer between the surface of the body and the fluid.

In many industrial applications including spacecraft boundary layer flow control, crystal growth, materials processing, metallic surface coating and MHD energy systems, the transport fluid is electrically-conducting and interacts with externally imposed magnetic fields. The Lorentz magnetic body force is used in such systems to control velocities and also reduce convection heat transfer rates. In the case of rotating scenarios, the centrifugal forces generated interact strongly with the magnetic forces and in the case of free convection with buoyancy forces. A number of researchers have studied hydromagnetic rotational convection flows, both from the viewpoint of internal flows and external boundary layer flows and applications include spacecraft heat transfer control $[15,16]$. Chamkha [17] considered the effects of wall mass transfer, heat generation, magnetism and porous resistances on MHD combined convection from a spinning cone. Takhar and Nath [18] obtained shooting solutions for transient MHD heat transfer in the stagnation region of a spinning sphere. Takhar et al. [19] extended this analysis to consider the supplementary effects of translation, buoyancy and impulsive motion on the MHD rotating sphere regime. Roy et al. [20] presented an excellent study of unsteady rotating cone MHD convection using finite difference methods. Unsteadiness was introduced into the model via a time-dependent angular velocity of the body and the fluid. Tangential and azimuthal shear stresses were shown to be significantly affected by magnetic field, surface velocity and also suction/injection effects. It was also shown that when the angular velocity of the fluid exceeds that of the rotating body, the velocity profiles attain asymptotic values in an oscillatory fashion at the boundary layer edge, and the magnetic field suppresses these oscillations. Takhar et al. [21] also studied the transient combined natural/forced MHD convection flow from a spinning cone. Ozturk [22] studied the mixed transient MHD convection from a rotating sphere. Periera and Sousa [23] studied vortex breakdown in rotating cone flow. Chamkha and Al Mudhaf [24] studied the combined MHD heat and mass transfer with heat sink effects in rotating cone convection. Abo-Eldahab and El Aziz [25] have studied micropolar rotating MHD cone convection with Hall, Joule heating and temperature power-law effects. Practically all these studies have considered only pure fluid regimes external to the rotating body. In numerous energy resources areas, however, the external medium is often porous. As such extra body forces must be incorporated into the analysis to account for linear porous resistance at lower Reynolds numbers. Porous media may also arise for example in 
debris-laden flow regimes surrounding spacecraft [26]. In certain plasma regimes (and also industrial ceramic foams and geomaterials), the medium also possesses anisotropic permeabilities i.e. the hydraulic conductivity of the medium is different in different directions. Such a property can have a substantial influence on momentum and heat transfer in the medium. Generally an anisotropic porous medium in an $(x, y, z)$ coordinate system, has a second order permeability tensor, $\boldsymbol{K}$, of the form, following Verruijt [27]:

$$
\boldsymbol{K}=\left[\begin{array}{lll}
K_{x x} & K_{x y} & K_{x z} \\
K_{y x} & K_{y y} & K_{y z} \\
K_{z x} & K_{z y} & K_{z z}
\end{array}\right]
$$

Generally the coefficients are not all unique so that the permeability tensor is symmetric in three-dimensional flows and it is therefore assumed that $K_{x y}=K_{y x}, K_{y z}=K_{z y}$ and $K_{z x}=$ $K_{x z}$. The permeability coefficients $K_{x x}, K_{y y}$ and $K_{z z}$ define the permeability in the $x, y$ and $z$ directions and therefore six values are generally needed to simulate a general threedimensional anisotropic porous medium. Clearly for the special case of isotropic flow all three permeabilities will be identical i.e. $K_{x x}=K_{y y}=K_{z z}$. For orthotropic porous media (with two mutually perpendicular and different permeabilities) three values are required. For two-dimensional porous orthotropic flow, as considered in this paper, we shall ignore the $z$-direction permeability. Orthotropic and more complex anisotropic flows have received some attention in fluid mechanics. For example Steck et al [28] studied anisotropic flows in biomaterials. Cheng [29] considered transport in anisotropic geomaterials. Most porous media heat transfer flow studies have also used the Darcy law. In the present study we shall therefore examine the hydromagnetic convection from a rotating cone to an orthotropic Darcian porous medium. The porous medium may simulate for example a filtration medium for controlling the spin chemical processing of materials. A Keller-Box numerical solution to the transformed partial differential equations is obtained.

\section{MATHEMATICAL MODEL}

The regime to be studied is illustrated below in Figure 1. The steady, laminar, incompressible, axisymmetric, hydromagnetic natural convection boundary layer flow along a rotating cone suspended in an orthoropic porous regime is considered. The cone is maintained at constant temperature (isothermal surface condition) as is the ambient fluid. The magnetic field is applied normal to the cone surface. The $X$ direction is parallel 
to the cone slant surface, the $Y$ direction normal to this and $\theta$ designates the angle in a plane perpendicular to the vertical symmetry axis. The cone may represent for example a chemical engineering mixing device. The governing equations for the electricallyconducting flow regime can be posed as follows with reference to an $(X, Y, \theta)$ co-ordinate system:

Conservation of Mass:

$$
\frac{\partial(R U)}{\partial X}+\frac{\partial(R V)}{\partial Y}=0
$$

Momentum

$$
U \frac{\partial U}{\partial X}+V \frac{\partial U}{\partial Y}-W^{2} \frac{(R)^{\prime}}{R}=v \frac{\partial^{2} U}{\partial Y^{2}}-\frac{v}{K_{X}} U+g \beta \operatorname{Cos} \phi\left(T-T_{\infty}\right)-\frac{\sigma B^{2}}{\rho} U
$$

\section{Momentum}

$$
U \frac{\partial W}{\partial X}+V \frac{\partial W}{\partial Y}+U W \frac{(R)^{\prime}}{R}=v \frac{\partial^{2} W}{\partial Y^{2}}-\frac{v}{K_{\theta}} W-\frac{\sigma B^{2}}{\rho} W
$$

Thermal Energy (Heat):

$$
U \frac{\partial T}{\partial X}+V \frac{\partial T}{\partial Y}=\alpha \frac{\partial^{2} T}{\partial Y^{2}}
$$

The Boussinesq approximation has been used so that buoyancy effects only appear in the $X$-direction momentum equation (3), which is coupled to the energy equation, constituting a free convection regime. Two separate hydromagnetic impedance terms are present in the momentum equations (3) and (4), each proportional to the velocity in the $X$ and $\theta$ direction respectively, with separate permeability parameters due to anisotropy. Also a porous resistance term is present in each of equation (3) and (4) for the Darcian impedance. Here $R$ is the dimensional radial coordinate and $(R)^{\prime}=$ dimensional radius of the cone. The magnetic Reynolds number is assumed to be small as there is negligible induced magnetic field compared with the applied magnetic field. Viscous dissipation and Ohmic heating (Joule electromagnetic dissipation) effects are also neglected. The corresponding boundary conditions at the surface and far from the cone are:

$$
\begin{aligned}
& U(X, 0)=V(X, 0)=0 ; W(X, 0)=0, T(X, 0)=T_{w} \\
& U(X, Y)=W(X, Y)=0 ; T(X, Y)=T_{\infty} \text { as } Y \rightarrow \infty .
\end{aligned}
$$


where all parameters are defined in the nomenclature. The equations (2) to (5) are highly coupled, parabolic and nonlinear. An analytical solution is clearly intractable and in order to obtain a robust solution, we next non-dimensionalize the model. This also serves the dual purpose of introducing dimensionless thermofluid parameters which provide an excellent mechanism for studying heat transfer and flow phenomena and circumvent the need for ascribing actual physical properties for the fluid.

\section{TRANSFORMATION OF MODEL}

We now introduce the following transformations to the model:

$$
\begin{aligned}
& x=\frac{X}{L}, y=\frac{Y}{L G r_{L}^{-1 / 4}}, r=\frac{R}{L}, u=\frac{U}{U *}, v=\frac{V}{U * G r_{L}^{-1 / 4}} . \\
& w=\frac{W}{\Omega L}, \kappa_{x}=\frac{K_{X}}{L^{2}}, \kappa_{\theta}=\frac{K_{\theta}}{L^{2}}, \operatorname{Pr}=\frac{v}{\alpha}, U^{*}=\left[g \operatorname{Cos} \phi \beta L\left(T_{w}-T_{\infty}\right)\right]^{1 / 2} . \\
& \Phi=\frac{T-T_{\infty}}{T_{w}-T_{\infty}}, G r_{L}=\left[\frac{U * L}{v}\right]^{2}, \operatorname{Re}=\frac{\Omega L^{2}}{v}, N m=\frac{\sigma B o^{2} L}{U * \rho} .
\end{aligned}
$$

where all parameters are defined in the nomenclature. The transport equations are thereby reduced to the following dimensionless partial differential equations:

Mass:

$$
\frac{\partial(r u)}{\partial x}+\frac{\partial(r v)}{\partial y}=0
$$

\section{Tangential Momentum}

$$
u \frac{\partial u}{\partial x}+v \frac{\partial u}{\partial y}-\frac{\operatorname{Re}^{2}}{G r} w^{2} / r=\frac{\partial^{2} u}{\partial y^{2}}-\frac{u}{\kappa_{x}}+\Phi-N m u
$$

Swirl Momentum

$$
u \frac{\partial w}{\partial x}+v \frac{\partial w}{\partial y}+u w / r=\frac{\partial^{2} w}{\partial y^{2}}-\frac{w}{\kappa_{\theta}}-N m w
$$

Thermal Energy (Heat):

$$
u \frac{\partial \Phi}{\partial x}+v \frac{\partial \Phi}{\partial y}=\frac{1}{\operatorname{Pr}} \frac{\partial^{2} \Phi}{\partial y^{2}}
$$

The boundary conditions are also transformed into:

$$
\begin{aligned}
& u(x, 0)=v(x, 0)=0 ; w(x, 0)=0, \Phi(x, 0)=1 \text { at } y=0 \\
& u(x, y)=w(x, y)=0 ; \Phi(x, y)=0 \text { as } y \rightarrow \infty .
\end{aligned}
$$


The non-dimensional equations (9) to (12) can be further simplified by employing appropriate similarity transformations. We first define a stream function, $\psi$, following Ece [30]:

$$
r u=\frac{\partial \psi}{\partial y}, r v=-\frac{\partial \psi}{\partial x}
$$

The boundary layer variables are now defined as follows, with $r=x \sin \phi$ :

$$
\psi(x, y)=x r F(y), w=r G(y), \Phi=x H(y)
$$

This leads readily to the following coupled system of "self-similar" ordinary differential equations:

\section{Tangential Momentum:}

$$
F^{\prime \prime \prime}+2 F F^{\prime \prime}-\left(F^{\prime}\right)^{2}-\left(\frac{1}{\kappa_{x}}+N m\right) F^{\prime}+\varepsilon G^{2}+H=0
$$

Swirl Momentum:

$$
G^{\prime \prime}+2 F G^{\prime}-2 F^{\prime} G-\left(\frac{1}{\kappa_{\theta}}+N m\right) G=0
$$

\section{Energy:}

$$
\frac{1}{\operatorname{Pr}} H^{\prime \prime}+2 F H^{\prime}-F^{\prime} H=0
$$

where $F$ is the boundary-layer stream function, $G$ is the boundary-layer rotational (swirl) velocity, $H$ is the boundary-layer temperature, $\kappa_{x}$ and $\kappa_{\theta}$ denote the $x$-direction and $\theta$ direction Darcy numbers, and $\varepsilon=\frac{(\operatorname{Re} \operatorname{Sin} \phi)^{2}}{G r}$ is the spin parameter. We note that for the purely fluid case, the functions $\kappa_{x} \rightarrow \infty$ and $\kappa_{\theta} \rightarrow \infty$ since the permeability of the orthotropic regime becomes infinite. In this scenario the solid fibers in the medium vanish. The self-similar momentum equations (17) and (18) then reduce to exactly the equations solved by Ece [30]:

$$
\begin{aligned}
& F^{\prime \prime \prime}+2 F F^{\prime \prime}-\left(F^{\prime}\right)^{2}-N m F^{\prime}+\varepsilon G^{2}+H=0 \\
& G^{\prime \prime}+2 F G^{\prime}-2 F^{\prime} G-N m G=0
\end{aligned}
$$


Equation (19) is identical also to the self- similar heat transfer equation solved by Ece [30]. The transformed boundary conditions are also identical to those solved by Ece [30] and for the current problem take the form:

$$
\begin{aligned}
& F(0)=0 ; F^{\prime}(0)=0 ; G(0)=1, H(0)=1 \text { at the cone surface }(y=0) \\
& F^{\prime}(y) \rightarrow 0 ; G(y) \rightarrow(0) ; H(y) \rightarrow 0 \text { in the free stream }(y \rightarrow \infty)
\end{aligned}
$$

\section{NUMERICAL SOLUTION BY KELLER-BOX METHOD}

The governing equations amount to a seventh order set of nonlinear, coupled ordinary differential equations with corresponding boundary conditions. Many numerical methods and codes are available for the solution of nonlinear magneto-hydrodynamic and multiphysical fluid dynamics problems. These include the finite element method [31], network simulation methodology [32], the local non-similarity method [33] and the CrankNicolson difference method [34] etc. Here we use the implicit and stable Keller box method, originally developed for aerodynamic boundary layer flows [35]. Essentially there are four fundamental steps intrinsic to the Keller box scheme:

\section{a) Reduction of Nth order partial differential equation system to $N 1$ st order equations \\ b) Finite Difference Discretization \\ c) Quasi-linearization of Non-Linear Keller Algebraic Equations \\ d) Block-tridiagonal Elimination of Linear Keller Algebraic Equations}

The nonlinear boundary layer equations are effectively reduced to a seventh order linear system which is solved by the block-elimination method. The numerical results are affected by the number of mesh points. Accurate results are produced by performing a mesh sensitivity analysis. The method has proved to be one of the most versatile for nonlinear multi-physical fluid dynamics simulations. Kumar et al. [36] used Keller's Box method to study the influence of vectored mass transfer with heat transfer on boundary layer flows with variable gas properties and non-unity Prandtl number. Daskalakis [37] studied steady incompressible laminar boundary layer flow along a vertical cylinder with isothermal walls in the mixed free and forced convection regime, using Keller's box method. Yih [38] investigated the effects of viscous dissipation and stress work on the hydromagnetic forced convection adjacent to a non-isothermal wedge, obtaining a numerical solution for the non-similar boundary layer equations with the Keller box method. Other studies include those by Bég et al. [39] on viscoelastic boundary layer 
thermal convection and Vasu et al. [40] on hydromagnetic boundary layers from spherical bodies in porous media. Further studies on non-linear convection flows modelled with Keller's box method include [41-44]. The Keller-Box method has been extensively validated and comparisons were made with the shooting quadrature solutions of Ece [30] for the purely fluid (infinite permeability case) and found to show excellent correlation, as shown in Tables 1 and 2. Details of the computational cell employed and discretization procedure associated with the Keller-Box scheme are provided in

\section{Appendix 1.}

\section{RESULTS AND DISCUSSION}

Extensive computations have been conducted to simulate the variation of the tangential velocity $\left(F^{\prime}\right)$, swirl velocity $(G)$ and temperature $(H)$ with distance, $y$, into the boundary layer (transverse to the cone surface); 5 key parameters are analyzed - Prandtl number $(\mathrm{Pr})$, magnetohydrodynamic number $(\mathrm{Nm}), x$-direction Darcy number $\left(\kappa_{x}\right), \theta$-direction Darcy number $\left(\kappa_{\theta}\right)$ and spin parameter $\left(\varepsilon=\frac{(\operatorname{Re} \operatorname{Sin} \phi)^{2}}{G r}\right)$. The regime has high permeability in both the $x$ - and $\theta$-directions so that high values are prescribed for $\kappa_{x}$ and $\kappa_{\theta}$. i.e. 0.3 , unless otherwise stated. $\operatorname{Pr}$ is prescribed as unity (accurate for gases), $\varepsilon=2.0$ and $N m=1$ (magnetohydrodynamic and viscous forces are the same order of magnitude), unless otherwise indicated. In the graphs $y$ is defined as the computational variable, $\eta$.

Figures 2 to 4 illustrate the influence of the $\kappa_{\theta}$ parameter on $F^{\prime}, G$ and $H$ profiles. An increase in the permeability function, $\kappa_{\theta}$ i.e. $\theta$-direction permeability acts to significantly elevate the tangential velocity close to the wall (figure 2); further from the cone surface however the effect is reversed and there is a slight decrease in tangential velocities as we progress further into the boundary layer. In close proximity to the wall, the decreased porous media drag caused by a reduction in the presence of porous media fibers in the $\theta$ direction (i.e. an increase in the Darcy parameter, $\kappa_{\theta}$ ) will act to accelerate the flow. Skin friction i.e. surface shear stress will therefore also be augmented considerably with increasing permeability function, $\kappa_{\theta}$.

Swirl velocity (figure 3) exhibits a very different response pattern; peak $G$ values always arise at the wall and decay smoothly to a minimum in the free stream. However the increase in $\kappa_{\theta}$ i.e. $\theta$-direction permeability $(=\theta$-direction Darcy parameter) consistently boosts the swirl velocity throughout the boundary layer (the flow is accelerated). 
Irrespective of the value of $\kappa_{\theta}$ there never arises any flow reversal in the boundary layer regime.

With increasing $\theta$-direction permeability temperatures (figure 4) are substantially reduced in the boundary layer regime. Increasing permeability decreases the concentration of solid particles in the regime i.e. increases the presence of voids. This serves to suppress thermal conduction heat transfer in the regime and acts to reduce temperatures. There will however be a corresponding increase in heat transferred to the cone surface with increasing values of tangential Darcy number $\left(\kappa_{\theta}\right)$. The influence of $\kappa_{\theta}$ on the temperature field is less prominent than on the tangential and swirl velocity fields, since permeability functions do not arise in the energy conservation equation (32); they feature as inverse functions in the momenta equations and are in fact related to the Darcian linear body forces impeding the flow (bulk matrix resistance at low Reynolds numbers). It is therefore expected that a change in $\kappa_{\theta}$ will modify tangential and swirl velocities to a much greater extent and indeed this is demonstrated by figures 2 and 3 . We further note that the swirl velocities are influenced more strongly by $\kappa_{\theta}$ than are the tangential velocities, since $\kappa_{\theta}$ arises only in the swirl momentum equation (31) and will, via coupling, only indirectly influence the tangential momentum equation (30) albeit weakly.

Figures 5 to 7 depict the evolution of tangential velocity, swirl velocity and temperature functions, with a variation in the $\kappa_{x}$ i.e. $x$-direction Darcy parameter. A significant acceleration in the tangential velocity is observed in figure 5; this effect is sustained to a much greater extent into the boundary layer than witnessed in figure 2 (for $\kappa_{\theta}$ ) variation. In the momentum equation (31) the $\kappa_{x}$ parameter arises in the Darcian retarding force term, $-F^{\prime} / \kappa_{x}$. Increasing $\kappa_{x}$ will act to decrease the Darcian drag force which will effectively accelerate the tangential flow. In figure 6 we note that very little change in the swirl velocity is computed with increasing the $\kappa_{x}$ parameter. The dominant influence is on the tangential flow (figure 5). Temperature, $H$, is however found to be strongly decreased with increasing $\kappa_{x}$. As indicated earlier with progressive increase in permeability, the regime comprises a lower quantity of solid material fibers; this suppresses thermal conduction and acts to cool the boundary layer.

The effect of the spin parameter, $\varepsilon$, on tangential velocity, swirl velocity and temperature functions, is presented in figures 8 to 10. In these figures, $\kappa_{x}=\kappa_{\theta}=0.3$ i.e. the regime is isotropic. $\varepsilon$ arises in eqn. (30), and the over-riding influence will be on the tangential 
momentum. With greater $\varepsilon$, the spin of the cone acts to significantly increase the magnitude of the tangential velocity profiles due to the induced axial flow, in particular close to the cone surface $(y=0)$; this pattern has been observed also by Ece [30]. Swirl velocity is however not significantly affected since the spin parameter does not arise in the swirl momentum equation (31). Temperature, $H$, is found to be reduced with increasing $\varepsilon$; this will simultaneously enhance the heat transfer rate to the cone surface.

Figures 11 to 13 show the tangential velocity, swirl velocity and temperature function profiles for variation in the hydromagnetic parameter $(\mathrm{Nm})$. The Lorentzian magnetic drag forces, $-N m F^{\prime}$ and $-N m G$ arising in the momenta equations (30) and (31), respectively, will exert a decelerating influence on the flow regime. In consistency with this we observe in both figures 11 and 12 that increasing $N m$ from 0 through 1, 2, 5 to 10 (strongest transverse magnetic field case) clearly suppresses both tangential and swirl velocities. Magnitudes of both velocity components are therefore maximized for the electrically non-conducting case $(\mathrm{Nm}=0)$. We note that for $M=1$ the viscous and hydromagnetic (Lorentzian) drag forces are of the same order; for $M>1$ hydromagnetic drag exceeds the viscous force and for $M<1$ vice versa. Velocity gradient normal to the surface, and, therefore, also wall shear stress both decrease with the magnetic parameter. Again a similar response has been computed by Ece [30]. Transverse magnetic field overall inhibits the flow development and serves as a powerful regulating mechanism in such regimes. Conversely temperature values $(H)$, as shown in figure 13 , are significantly elevated with increasing values of the magnetic parameter. The supplementary work done in dragging the fluid against the action of the magnetic field in the porous medium is dissipated as thermal energy; this therefore acts to heat the thermal boundary layer and increases temperatures. As a result the surface heat flux on the cone considerably decreases with an increase in $\mathrm{Nm}$. We refer not to viscous dissipation here, but supplementary work done which is expended as heat in magnetohydrodynamics. This is not viscous dissipation since viscous dissipation can arise without a magnetic field. The thermal effect associated with magnetohydrodynamic flow has been confirmed experimentally as early as 1958 by NASA researchers [45]. Dragging a fluid against the inhibitive Lorentzian magnetic drag generates heat via a different mechanism than that associated with enthalpy changes in high speed flows where viscous heating arises [46]. Viscous heating is usually simulated via the Eckert number. No such parameter is featured in our model since we are not studying high speed (inertial-dominated) aerodynamics, but very low speed viscous dominated heat transfer. At such low speeds, 
Eckert effects do not arise. Effectively with greater magnetic field the cone surface is cooled and the thermal boundary layer is heated.

Figures 14 to 16 present the influence of Prandtl number (Pr) on the velocity functions and temperature field. Larger $\operatorname{Pr}$ values (e.g. $\operatorname{Pr}=10$, corresponds to certain oils and lubricants) correspond to a thinner thermal boundary layer thickness and more uniform temperature distributions across the boundary layer. Smaller $\operatorname{Pr}$ fluids (e.g. $\operatorname{Pr}=0.01$ corresponds to liquid metal) possess higher thermal conductivities so that heat can diffuse away from the cone surface faster than for higher Pr fluids (low Pr fluids correspond to thicker boundary layers). $\operatorname{Pr}$ defines the ratio of momentum diffusivity to thermal diffusivity for a given fluid implying that for lower $\operatorname{Pr}$ fluids, heat diffuses faster than momentum and vice versa for higher $\operatorname{Pr}$ fluids. For $\operatorname{Pr}=1$ the momentum and thermal boundary layers are of the same thickness. For $\operatorname{Pr}>>1$, (e.g. 10) the thermal boundary layer is embedded in the momentum boundary layer since the Prandtl number is much larger than unity and free convection effects are damped thereby contributing less to driving the fluid motion.

\begin{tabular}{llllll}
\hline$\varepsilon$ & $M$ & \multicolumn{5}{c}{$\mathrm{F}^{\prime \prime}(0)$} \\
\hline \multirow{2}{*}{0.0} & 0 & 0.68150212 & 0.68150211 & 0.43327726 & 0.43327724 \\
& 1 & 0.55975901 & 0.55975900 & 0.37927751 & 0.37927749 \\
& 2 & 0.48678916 & 0.48678914 & 0.34866381 & 0.34866378 \\
& 5 & 0.37090743 & 0.37090742 & 0.29345341 & 0.29345340 \\
& 10 & 0.28500344 & 0.28500343 & 0.24343322 & 0.24343321 \\
0.5 & 0 & 0.84650616 & 0.84650614 & 0.62601869 & 0.62601867 \\
& 1 & 0.68547905 & 0.68547904 & 0.50300655 & 0.50300654 \\
& 2 & 0.59002983 & 0.59002983 & 0.44721675 & 0.44721673 \\
& 5 & 0.44274371 & 0.44274370 & 0.36247167 & 0.36247166 \\
1.0 & 10 & 0.33709053 & 0.33709051 & 0.29437077 & 0.29437075 \\
& 0 & 1.00196008 & 1.00196009 & 0.79828572 & 0.79828571 \\
& 1 & 0.80819380 & 0.80819378 & 0.62583066 & 0.62583065 \\
& 2 & 0.69203710 & 0.69203709 & 0.54556916 & 0.54556916 \\
& 5 & 0.51435738 & 0.51435736 & 0.43140288 & 0.43140286 \\
2.0 & 10 & 0.38913125 & 0.38913123 & 0.34527676 & 0.34527674 \\
& 0 & 1.29230021 & 1.29230020 & 1.10990481 & 1.10990480 \\
& 1 & 1.04586353 & 1.04586352 & 0.86705752 & 0.86705750 \\
& 0.89263261 & 0.89263260 & 0.74142462 & 0.74142460 \\
& 5 & 0.65693141 & 0.65693140 & 0.56901873 & 0.56901871 \\
& 10 & 0.49307448 & 0.49307445 & 0.44699636 & 0.44699634 \\
\hline
\end{tabular}

Table 1: Values of $\mathrm{F}^{\prime \prime}(0)$ for free-convection boundary-layer flow over a spinning cone with $\kappa_{x} \rightarrow \infty$ and $\kappa_{\theta} \rightarrow \infty$ (purely fluid case) 


\begin{tabular}{llllll}
\hline$\varepsilon$ & $M$ & \multicolumn{5}{c}{$-\mathrm{H}^{\prime}(0)$} & & \\
\cline { 3 - 6 } 0.0 & 0 & 0.63886614 & 0.63886613 & 1.27552680 & 1.27552678 \\
& 1 & 0.55869398 & 0.55869396 & 1.16243080 & 1.16243079 \\
& 2 & 0.50338419 & 0.50338417 & 1.09342644 & 1.09342642 \\
& 5 & 0.40364781 & 0.40364781 & 0.95839216 & 0.95839215 \\
& 10 & 0.32121113 & 0.32121112 & 0.82300420 & 0.82300418 \\
0.5 & 0 & 0.67194897 & 0.67194895 & 1.47165986 & 1.47165984 \\
& 1 & 0.58138075 & 0.58138073 & 1.27668867 & 1.27668866 \\
& 2 & 0.51966708 & 0.51966708 & 1.17427021 & 1.17427020 \\
& 5 & 0.41156185 & 0.41156183 & 1.00202092 & 1.00202091 \\
& 10 & 0.32496333 & 0.32496332 & 0.84700807 & 0.84700805 \\
& 0 & 0.70053401 & 0.70053400 & 1.60768499 & 1.60768498 \\
& 1 & 0.60256486 & 0.60256484 & 1.37917914 & 1.37917912 \\
& 2 & 0.53536409 & 0.53536408 & 1.25082089 & 1.25082087 \\
& 5 & 0.41940005 & 0.41940002 & 1.04490075 & 1.04490072 \\
& 10 & 0.32870765 & 0.32870761 & 0.87088877 & 0.87088872 \\
& 0 & 0.74869559 & 0.74869555 & 1.80575019 & 1.80575017 \\
& 1 & 0.64120157 & 0.64120154 & 1.55413661 & 1.55413658 \\
& 2 & 0.56516379 & 0.56516378 & 1.39136129 & 1.39136127 \\
& 5 & 0.43485365 & 0.43485362 & 1.12833102 & 1.12833101 \\
& 10 & 0.33617261 & 0.33617259 & 0.91826049 & 0.91826045 \\
\hline
\end{tabular}

Table 2: Values of $-\mathrm{H}^{\prime}(0)$ for free-convection boundary-layer flow over a spinning cone with $\kappa_{x} \rightarrow \infty$ and $\kappa_{\theta} \rightarrow \infty$ (purely fluid case)

With increasing $P r$, the tangential velocity (figure 14) is very strongly decelerated; swirl velocity (figure 15) is however practically unaffected. Temperature, $H$, as expected, is markedly suppressed throughout the boundary layer regime (figure 16), with an increase in $\operatorname{Pr}$. Maximum $H$ corresponds to the lowest $\operatorname{Pr}$ value (0.01 i.e. liquid metals) since thermal conductivities are much higher for such cases.

\section{CONCLUSIONS}

A mathematical model has been developed for the magnetohydrodynamic free convection flow of a spinning cone embedded in an orthotropic Darcian highly permeable medium. The governing equations have been transformed into self-similar form and solved under physically realistic boundary conditions using the Keller Box finite difference method. Validation with previous studies has been included. Increasing magnetic field has been shown to generally decelerate the tangential and swirl flow and accentuate temperatures. Tangential and swirl velocities have also been found to be generally elevated with 
increasing $x$ - and $\theta$-direction permeability functions (i.e. Darcy parameters) owing to a corresponding reduction in the Darcian body forces. Increasing spin of the cone has been shown to boost the tangential velocities but exerts negligible effect on the swirl velocity. The problem considered is steady-state and Newtonian and considers only heat transfer. Future studies will examine unsteady non-Newtonian flows with mass transfer [47].

\section{ACKNOWLEDGEMENTS}

The authors are grateful to both reviewers for their careful appraisals of the work which has served to improve the clarity and quality of the final paper.

\section{REFERENCES}

[1] Wu, C., The three-dimensional incompressible laminar boundary layer on a spinning cone, Appl. Sci. Res., 8, 140 (1959).

[2] Hering, R.G. and Grosh, R.J., Laminar combined convection from a rotating cone, ASME J. Heat Transfer, 85, 29 (1963).

[3] Paterson, J.A., Grief, R. and Cornet, I., Experimental and theoretical results for mass transfer to a rotating cone in non-Newtonian solutions, Int. J. Heat and Mass Transfer, 16, 1017-1024 (1973).

[4] Smith, R.N. and Grief, R., Laminar convection to rotating cones and disks in nonNewtonian power-law fluids, Int. J. Heat and Mass Transfer, 18, 1249-1253 (1975).

[5] Jeng, D.R. and Chao, B.T., Unsteady heat transfer from a rotating disk or cone with large suction, 25 $5^{\text {th }}$ Heat Transfer and Fluid Mechanics Institute, USA (1976).

[6] Himasekhar, K. and Sarma, P.K., Laminar combined convection from a rotating cone to a thermally stratified environment, ASME J. Heat Transfer, 108, 973 (1986).

[7] Lin, H.T. and Lin, L.K., Heat transfer from a rotating cone or disk to fluids of any Prandtl number, Int. Comm. Heat and Mass Transfer, 14, 323 (1987).

[8] Wang, T.Y. and Kleinstreuer, C., Similarity solutions of combined convection heat transfer from a rotating cone or disk to non-Newtonian fluids, ASME J. Heat Transfer, 112, 939 (1990).

[9] Gorla, R.S.R. and Nakamura , S., Mixed convection of a micropolar fluid from a rotating cone, Int. J. Heat and Fluid Flow, 16, $69-73$ (1995). 
[10] Mao, T-S. and Wang, Y.C., Holographic visualization of convective flow around a heated rotating cone of finite length, ASME J. Fluids Engineering, 115, 3, 515-522 (1993).

[11] Chen, H., Jebson, R.S. and Campanella, O.H., Determination of heat transfer coefficients in rotating cone evaporators: Part 1, Proc. IChemE : Foods and Bioproducts Processing, 75, C1, 17-22 (1997).

[12] Venkateswarlu, P., Babu, D.J., Naidu, S.V. and Subba Rao, D., Studies on ionic mass transfer at a rotating cone in an open cell, Int. Comm. Heat and Mass Transfer, 31, 2, 221-230 (2004).

[13] Anilkumar, D. and Roy, S., Self-similar solution to unsteady mixed convection flow from a rotating cone in a rotating fluid, SIAM Conf. Applied Mathematics, Florida, Gainsville, USA, 3-4 March (2004).

[14] Ozturk, A. and Ece, M.C., Unsteady forced convection heat transfer from a translating and spinning body, ASME J. Energy Res. Tech., 117, 318-323 (1995).

[15] Macheret, S.O., Shneider, M.N., and Miles, R.B., Magnetohydrodynamic and electrohydrodynamic control of hypersonic flows of weakly ionized plasmas, AIAA J., 42, 7, 1378-1387 (2004).

[16] Keidar, M., Kim, M., and Boyd, I.D., Electromagnetic reduction of plasma density during atmospheric reentry and hypersonic flights, J. Spacecraft and Rockets, 45, 445453 (2008).

[17] Chamkha, A.J., Magnetohydrodynamic mixed convection from a rotating cone embedded in a porous medium with heat generation, J. Porous Media, 2 (1999).

[18] Takhar, H.S. and Nath, G., Self-similar solution of the unsteady flow in the stagnation point region of a rotating sphere with a magnetic field, Heat and Mass Transfer J., 36, 89-96 (2000).

[19] Takhar, H.S., Chamkha, A.J. and Nath, G., Unsteady laminar MHD flow and heat transfer in the stagnation region of an impulsively spinning and translating sphere in the presence of buoyancy forces, Heat and Mass Transfer J., 37, 397-402 (2001).

[20] Roy, S., Takhar, H.S. and Nath, G., Unsteady MHD flow on a rotating cone in a rotating fluid, Acta Mechanica, 150, 1/2, 67-77 (2002).

[21] Takhar, H.S., Chamkha, A.J. and Nath, G., Unsteady mixed convection flow from a rotating vertical cone with magnetic field, Heat and Mass Transfer J., 39, 297-304 (2003). 
[22] Ozturk, A., Unsteady laminar mixed convection about a spinning sphere with a magnetic field, Heat and Mass Transfer J., 41, 864-874 (2005).

[23] Periera, J.C.F. and Sousa, J.M.M., Confined vortex breakdown generated by a rotating cone, J. Fluid Mechanics, 385, 287-323 (1999).

[24] Chamkha, A.J. and Al-Mudhaf, Unsteady heat and mass transfer from a rotating vertical cone with a magnetic field and heat generation or absorption effects, Int. J. Thermal Sciences, 44, 267-276 (2005).

[25] Abo-Eldahab, E.M. and El Aziz, M.A., Hall current and Ohmic heating effects on mixed convection boundary layer flow of a micropolar fluid from a rotating cone with power-law variation in surface-temperature, Int. Comm. Heat Mass Transfer, 31, 751-762 (2004).

[26] Zueco, J. and O. Anwar Bég, Network simulation solutions for laminar radiating dissipative magneto-gas dynamic heat transfer over a wedge in non-Darcian porous regime, Mathematical and Computer Modelling, 50, 439-452 (2009).

[27] Verruijt, A., Groundwater Flow, second edition, Macmillan Press, UK (1982).

[28] Steck, R., Niederer, P and Knothe Tate, M.L., Fluid flows through anisotropic poroelastic bone models in the opposite direction to that through analogous isotropic models, Summer Bioengineering Conf., June 25-29, Key Biscayne, Florida, USA (2003).

[29] Cheng, A.H.D., Material coefficients of anisotropic poroelasticity, Int. J. Rock Mech. Mining Sci., 34, 199-205 (1997).

[30] Ece, M.C., Free convection flow about a vertical spinning cone under a magnetic field, Applied Mathematics and Computation, 179, 231-242 (2006).

[31] Bég, O. Anwar, H. S. Takhar, R. Bharagava, Rawat, S. and Prasad, V.R., Numerical study of heat transfer of a third grade viscoelastic fluid in non-Darcian porous media with thermophysical effects, Physica Scripta, 77, 1-11 (2008).

[32] Bég, O. Anwar, H. S. Takhar, Joaquín Zueco, A. Sajid and R. Bhargava, Transient Couette flow in a rotating non-Darcian porous medium parallel plate configuration: network simulation method solutions, Acta Mechanica, 200, 129-144 (2008).

[33] Bég, O. Anwar, Bakier, A.Y. and V.R. Prasad, Numerical study of free convection magnetohydrodynamic heat and mass transfer from a stretching surface to a saturated porous medium with Soret and Dufour effects, Computational Materials Science, 46, 1, 57-65 (2009). 
[34] Mohiddin, S.G., V. R. Prasad, S. V. K. Varma and O. Anwar Bég, Numerical study of unsteady free convective heat and mass transfer in a Walters-B viscoelastic flow along a vertical cone, Int. J. Applied Mathematics and Mechanics, 6, 88-114 (2010).

[35] Keller, H.B., Numerical methods in boundary-layer theory, Annual Review of Fluid Mechanics, 10, 417-433 (1978).

[36] Kumar, P., P. Sambath Narayanan and S. Raut, Laminar boundary layers with vectored mass transfer, Int. J. Engineering Science, 25, 1503-1509 (1987).

[37] Daskalakis, J. E. Mixed free and forced convection in the incompressible boundary layer along a rotating vertical cylinder with fluid injection. Int. J. Energy Research, 17: 689-695 (1993).

[38] Yih, K.A., MHD forced convection flow adjacent to a non-isothermal wedge, Int. Comm. Heat and Mass Transfer, 26, 819-827 (1999).

[39] O. Anwar Bég, Tasveer A. Bég, H S. Takhar and A. Raptis, Mathematical and numerical modeling of non-Newtonian thermo-hydrodynamic flow in non-Darcy porous media, Int. J. Fluid Mechanics Research, 31, 1-12 (2004).

[40] Vasu, B., V. R. Prasad, O. Anwar Bég, A. Aziz, and Rana D. Prashad, Numerical analysis of magnetohydrodynamic nonlinear convection heat and mass transfer from a sphere in a non-Darcian variable-porosity medium, Int. J. Applied Mathematics and Mechanics, 6, 64 - 111 (2010).

[41] V. R. Prasad, S. A. Gaffar and O. Anwar Bég, Heat and mass transfer of a nanofluid from a horizontal cylinder to a micropolar fluid, AIAA J. Thermophysics Heat Transfer (2014). 13 pages. DOI: 10.2514/1.T4396.

[42] V. R. Prasad, S. Abdul Gaffar, E. Keshava Reddy and O. Anwar Bég, Computational study of non-Newtonian thermal convection from a vertical porous plate in a non-Darcy porous medium with Biot number effects, J. Porous Media, 17 (7) (2014).

[43] S.Abdul Gaffar, V.R. Prasad and O. Anwar Bég, Flow and heat transfer of Jefferys non-Newtonian fluid from a horizontal circular cylinder, AIAA J. Thermophysics and Heat Transfer (2014). doi: 10.2514/1.T4253

[44] V.R. Prasad, S. Abdul Gaffar, E. Keshava Reddy and O. Anwar Bég, Numerical study of non-Newtonian boundary layer flow of Jeffreys fluid past a vertical porous plate in a non-Darcy porous medium, Int. J. Comp. Meth. Engineering Science \& Mechanics, 15 (4) 372-389 (2014).

[45] V.J. Rossow, On the flow of electrically-conducting fluids over a flat plate in the presence of a transverse magnetic field, NACA Report, USA 1358 (1958). 
[46]K. C. Cramer, Several magneto hydrodynamic free convection solutions, ASME J Heat Transfer, 26, 35-40 (1963).

[47] TB Chang, A Mehmood, O. Anwar Bég, M Narahari, MN Islam, F Ameen, Numerical study of transient free convective mass transfer in a Walters-B viscoelastic flow with wall suction, Communications in Nonlinear Science and Numerical Simulation, 16 (1), 216-225 (2011). 


\section{APPENDIX 1: KELLER-BOX FINITE DIFFERENCE SCHEME}

A 2-dimensional computational grid is imposed on the transformed boundary layer domain as shown in Fig A1. Note that since only one independent variable $(\eta)$ is employed, the streamwise variable $(\xi)$ is not discretized in the numerical computations.

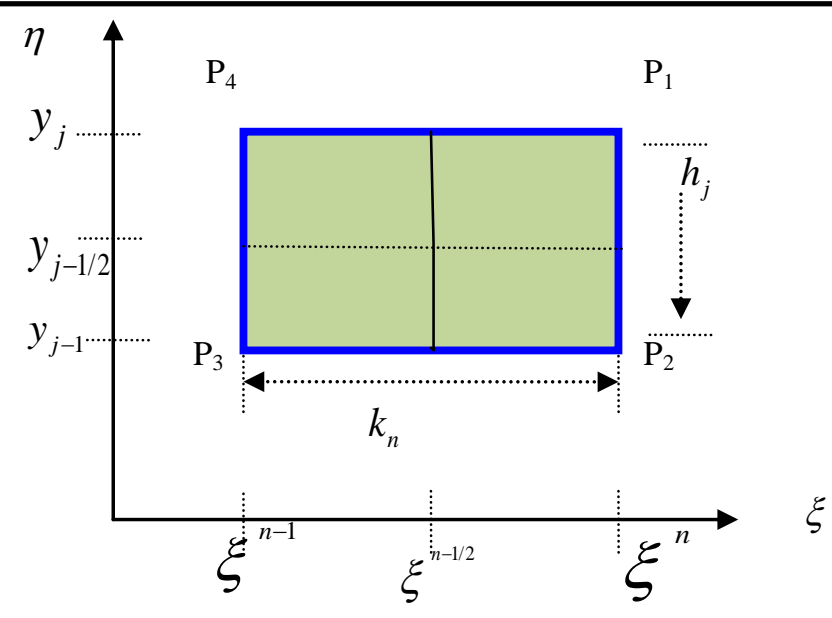

Figure A1. "Keller-Box" computational cell for finite difference approximation

The numerical stepping process is defined only for the $\eta$-coordinate by:

$$
y_{0}=0 ; y_{j}=y_{j-1}+h_{j}, j=1,2 \ldots J
$$

where $h_{j}$ denote the step distance in the $y$ direction. Denoting $\Sigma$ as the value of any variable at station $y_{j}$, and the following central difference approximations are substituted for each reduced variable and their first order derivatives, viz:

$$
\begin{aligned}
& (\Sigma)^{\mathrm{n}-1 / 2}{ }_{\mathrm{j}-1 / 2}=\left[\Sigma^{\mathrm{n}}{ }_{\mathrm{j}}+\Sigma^{\mathrm{n}}{ }_{\mathrm{j}-1}+\Sigma^{\mathrm{n}-1}{ }_{\mathrm{j}}+\Sigma^{\mathrm{n}-1}{ }_{\mathrm{j}-1}\right] / 4 \\
& (\partial \Sigma / \partial y)^{n-1 / 2}{ }_{j-1 / 2}=\left[\Sigma^{n}{ }_{j}+\Sigma^{n}{ }_{j-1}-\Sigma^{n-1}{ }_{j}-\Sigma^{n-1}{ }_{j-1}\right] / 4 h_{j}
\end{aligned}
$$

where $h_{j}=$ spanwise stepping distance ( $y$-mesh spacing) defined as follows:

$$
y_{j-1 / 2}=\left[y_{j}+y_{j-1}\right] / 2
$$

Phase a) Reduction of the $N^{\text {th }}$ order partial differential equation system to $N X 1^{\text {st }}$ order equations

Equations (30) to (32) subject to the boundary conditions (35) constitute a $7^{\text {th }}$ order wellposed two-point boundary value problem. The Eqns. (30) to (32) are first written as a 
system of 7 first-order equations. For this purpose, we introduce new dependent variables $u(y), v(y), t(y)$ and $p(y)$. Therefore, we obtain the following seven first-order equations:

$$
\begin{aligned}
& F^{\prime}=u \\
& u^{\prime}=v \\
& G^{\prime}=t \\
& H^{\prime}=p \\
& v^{\prime}+2 F v-u^{2}-\left(\frac{1}{\kappa_{x}}+N m\right) u+\varepsilon G^{2}+H=0 \\
& t^{\prime}+2 F t-2 u G-\left(\frac{1}{\kappa_{\theta}}+N m\right) G=0 \\
& \frac{1}{\operatorname{Pr}} p^{\prime}+2 F p-u H=0
\end{aligned}
$$

where primes denote differentiation with respect to $\eta$. In terms of the dependent variables, the boundary conditions become:

$$
\begin{array}{ll}
F=0, & u=0, \quad G=1, \quad H=1 \text { at } \quad y=0 \\
u \rightarrow 0, & G \rightarrow 0, \quad H \rightarrow 0 \quad \text { as } \quad y \rightarrow \infty
\end{array}
$$

\section{Phase b) Finite Difference Discretization}

The net points are denoted by:

$$
y_{0}=0, \quad y_{j}=y_{j-1}+h_{j}, \quad j=1,2, \ldots . J, \quad y_{J} \equiv y_{\infty},
$$

where $h_{j}$ is the $\Delta \eta$-spacing. Here $j$ is just sequence number that indicate the coordinate location. We approximate the quantities $(F, u, v, G, t, H, p)$ at points $\left(y_{j}\right)$ of the net by $\left(F_{j}^{n}, u_{j}^{n}, v_{j}^{n}, G_{j}^{n}, t_{j}^{n}, H_{j}^{n}, p_{j}^{n}\right)$, which we denote as net functions. We also employ the notion ( $)_{j}^{n}$ for points and quantities midway between net points and for any net function:

$$
y_{j-1 / 2} \equiv \frac{1}{2}\left(y_{j}+y_{j-1}\right),()_{j}^{n-1 / 2}=\frac{1}{2}\left[()_{j}^{n}+()_{j}^{n-1}\right] \text { and }()_{j-1 / 2}^{n}=\frac{1}{2}\left[()_{j}^{n}+()_{j-1}^{n}\right]
$$


We start by writing the finite-difference approximations of the ordinary differential equations A4 to A7 using centered-difference derivatives. This process is called "centering about $\left(y_{j-1 / 2}\right)$ ". This gives:

$$
\begin{aligned}
& \frac{\left(F_{j}^{n}-F_{j-1}^{n}\right)}{h_{j}}=\frac{1}{2}\left(u_{j}^{n}+u_{j-1}^{n}\right)=u_{j-1 / 2}^{n}, \\
& \frac{\left(u_{j}^{n}-u_{j-1}^{n}\right)}{h_{j}}=\frac{1}{2}\left(v_{j}^{n}+v_{j-1}^{n}\right)=v_{j-1 / 2}^{n}, \\
& \frac{\left(G_{j}^{n}-G_{j-1}^{n}\right)}{h_{j}}=\frac{1}{2}\left(t_{j}^{n}+t_{j-1}^{n}\right)=t_{j-1 / 2}^{n}, \\
& \frac{\left(H_{j}^{n}-H_{j-1}^{n}\right)}{h_{j}}=\frac{1}{2}\left(p_{j}^{n}+p_{j-1}^{n}\right)=p_{j-1 / 2}^{n},
\end{aligned}
$$

The differential equations (A8) to (A10) take the form:

$$
\begin{aligned}
& \left(v^{\prime}\right)^{n}+2(F v)^{n}-\left(u^{2}\right)^{n}-\left(\frac{1}{\kappa_{x}}+N m\right) u^{n}+\varepsilon\left(G^{2}\right)^{n}+H^{n} \\
& =-\left[\left(v^{\prime}\right)+2(F v)-\left(u^{2}\right)-\left(\frac{1}{\kappa_{x}}+N m\right) u+\varepsilon\left(G^{2}\right)+H\right]^{n-1} \\
& \left(t^{\prime}\right)^{n}+2(F t)^{n}-2(u G)^{n}-\left(\frac{1}{\kappa_{\theta}}+N m\right) G^{n}=-\left[\left(t^{\prime}\right)+2(F t)-2(u G)-\left(\frac{1}{\kappa_{\theta}}+N m\right) G\right]^{n-1} \\
& \frac{1}{\operatorname{Pr}}\left(p^{\prime}\right)^{n}+2(F p)^{n}-(u H)^{n}=\left[\frac{1}{\operatorname{Pr}}\left(p^{\prime}\right)+2(F p)-(u H)\right]^{n-1}
\end{aligned}
$$

where the notation []$^{n-1}$ corresponds to quantities in the square bracket evaluated at $y=y^{n-1}$. Discretization gives: 


$$
\begin{aligned}
& \left(\frac{v_{j}^{n}-v_{j-1}^{n}}{h_{j}}\right)+2\left(F_{j-1 / 2}^{n} v_{j-1 / 2}^{n}\right)-\left(u_{j-1 / 2}^{n}\right)^{2}-\left(\frac{1}{\kappa_{x}}+N m\right) u_{j-1 / 2}^{n}+\varepsilon\left(G_{j-1 / 2}^{n}\right)^{2}+H_{j-1 / 2}^{n} \\
& =-\left[\left(\frac{v_{j}^{n-1}-v_{j-1}^{n-1}}{h_{j}}\right)+2\left(F_{j-1 / 2}^{n-1} v_{j-1 / 2}^{n-1}\right)-\left(u_{j-1 / 2}^{n-1}\right)^{2}-\left(\frac{1}{\kappa_{x}}+N m\right) u_{j-1 / 2}^{n-1}+\varepsilon\left(G_{j-1 / 2}^{n-1}\right)^{2}+H_{j-1 / 2}^{n-1}\right] \\
& \begin{aligned}
\left(\frac{t_{j}^{n}-t_{j-1}^{n}}{h_{j}}\right)+2\left(F_{j-1 / 2}^{n} t_{j-1 / 2}^{n}\right)-2\left(u_{j-1 / 2}^{n} G_{j-1 / 2}^{n}\right)-\left(\frac{1}{\kappa_{\theta}}+N m\right) G_{j-1 / 2}^{n} & \\
= & -\left[\left(\frac{t_{j}^{n-1}-t_{j-1}^{n-1}}{h_{j}}\right)+2\left(F_{j-1 / 2}^{n-1} t_{j-1 / 2}^{n-1}\right)-2\left(u_{j-1 / 2}^{n-1} G_{j-1 / 2}^{n-1}\right)-\left(\frac{1}{\kappa_{\theta}}+N m\right) G_{j-1 / 2}^{n-1}\right]
\end{aligned} \\
& \frac{1}{\operatorname{Pr}}\left(\frac{p_{j}^{n}-p_{j-1}^{n}}{h_{j}}\right)+2\left(F_{j-1 / 2}^{n} p_{j-1 / 2}^{n}\right)-\left(u_{j-1 / 2}^{n} H_{j-1 / 2}^{n}\right) \\
& =-\left[\frac{1}{\operatorname{Pr}}\left(\frac{p_{j}^{n-1}-p_{j-1}^{n-1}}{h_{j}}\right)+2\left(F_{j-1 / 2}^{n-1} p_{j-1 / 2}^{n-1}\right)-\left(u_{j-1 / 2}^{n-1} H_{j-1 / 2}^{n-1}\right)\right]
\end{aligned}
$$

Equations (A21) and (A23) are imposed for $j=1,2 \ldots J$ at given $n$, and the transformed boundary layer thickness, $y_{J}$ is to be sufficiently large so that it is beyond the edge of the boundary layer.

$$
F_{0}^{n}=u_{0}^{n}=0, \quad G_{0}^{n}=1, \quad H_{0}^{n}=1, \quad u_{J}^{n}=0, \quad G_{J}^{n}=0, \quad H_{J}^{n}=0
$$

\section{Phase c) Quasi-linearization of Non-Linear Keller Algebraic Equations}

Newton's Method is then employed to quasi-linearize the equations (A21) to (A23). If we assume $\left(F_{j}^{n-1}, u_{j}^{n-1}, v_{j}^{n-1}, G_{j}^{n-1}, t_{j}^{n-1}, H_{j}^{n-1}, p_{j}^{n-1}\right)$ to be know for $0 \leq j \leq J$, then equations (A14) to (A17) and (A21) to (A23) with (A24) are a system of equations for the solution of the unknowns $\left(F_{j}^{n}, u_{j}^{n}, v_{j}^{n}, G_{j}^{n}, t_{j}^{n}, H_{j}^{n}, p_{j}^{n}\right), j=0,1,2, \ldots . . J$. For simplicity of notation we shall write the unknowns at $y=y^{n}$ as: 


$$
\left(F_{j}^{n}, u_{j}^{n}, v_{j}^{n}, G_{j}^{n}, t_{j}^{n}, H_{j}^{n}, p_{j}^{n}\right) \equiv\left(F_{j}, u_{j}, v_{j}, G_{j}, t_{j}, H_{j}, p_{j}\right)
$$

Then the system of equations considered then reduces to (after multiplying with $h_{j}$ ):

$$
\begin{aligned}
& F_{j}-F_{j-1}-\frac{h_{j}}{2}\left(u_{j}+u_{j-1}\right)=0 \\
& u_{j}-u_{j-1}-\frac{h_{j}}{2}\left(v_{j}+v_{j-1}\right)=0 \\
& G_{j}-G_{j-1}-\frac{h_{j}}{2}\left(t_{j}+t_{j-1}\right)=0 \\
& H_{j}-H_{j-1}-\frac{h_{j}}{2}\left(p_{j}+p_{j-1}\right)=0 \\
& \left(v_{j}-v_{j-1}\right)+\frac{h_{j}}{2}\left[\left(F_{j}+F_{j-1}\right)\left(v_{j}+v_{j-1}\right)\right]-\frac{h_{j}}{4}\left(u_{j}+u_{j-1}\right)^{2} \\
& -\frac{h_{j}}{2}\left(\frac{1}{\kappa_{x}}+N m\right)\left(u_{j}+u_{j-1}\right)+\frac{\varepsilon h_{j}}{4}\left(G_{j}+G_{j-1}\right)^{2}+\frac{h_{j}}{2}\left(H_{j}+H_{j-1}\right)=\left[R_{1}\right]_{j-1 / 2}^{n-1} \\
& \left(t_{j}-t_{j-1}\right)+\frac{h_{j}}{2}\left[\left(F_{j}+F_{j-1}\right)\left(t_{j}+t_{j-1}\right)\right]-\frac{h_{j}}{2}\left[\left(u_{j}+u_{j-1}\right)\left(G_{j}+G_{j-1}\right)\right] \\
& -\left(\frac{1}{\kappa_{\theta}}+N m\right) \frac{h_{j}}{2}\left(u_{j}+u_{j-1}\right)=\left[R_{2}\right]_{j-1 / 2}^{n-1} \\
& \frac{1}{\operatorname{Pr}}\left(p_{j}-p_{j-1}\right)+\frac{h_{j}}{2}\left[\left(F_{j}+F_{j-1}\right)\left(p_{j}+p_{j-1}\right)\right]-\frac{h_{j}}{4}\left[\left(u_{j}+u_{j-1}\right)\left(H_{j}+H_{j-1}\right)\right]=\left[R_{3}\right]_{j-1 / 2}^{n-1}
\end{aligned}
$$

where:

$$
\begin{aligned}
& {\left[R_{1}\right]_{j-1 / 2}^{n-1}=-h_{j}\left[\left(\frac{v_{j}-v_{j-1}}{h_{j}}\right)+2\left(F_{j-1 / 2} v_{j-1 / 2}\right)-\left(u_{j-1 / 2}\right)^{2}-\left(\frac{1}{\kappa_{x}}+N m\right) u_{j-1 / 2}+\varepsilon\left(G_{j-1 / 2}\right)^{2}+H_{j-1 / 2}\right]} \\
& {\left[R_{2}\right]_{j-1 / 2}^{n-1}=-h_{j}\left[\left(\frac{t_{j}-t_{j-1}}{h_{j}}\right)+2\left(F_{j-1 / 2} t_{j-1 / 2}\right)-2\left(u_{j-1 / 2} G_{j-1 / 2}\right)-\left(\frac{1}{\kappa_{\theta}}+N m\right) G_{j-1 / 2}\right]} \\
& {\left[R_{3}\right]_{j-1 / 2}^{n-1}=-h_{j}\left[\frac{1}{\operatorname{Pr}}\left(\frac{p_{j}-p_{j-1}}{h_{j}}\right)+2\left(F_{j-1 / 2} p_{j-1 / 2}\right)-\left(u_{j-1 / 2} H_{j-1 / 2}\right)\right]}
\end{aligned}
$$


$\left[R_{1}\right]_{j-1 / 2}^{n-1},\left[R_{2}\right]_{j-1 / 2}^{n-1}$ and $\left[R_{3}\right]_{j-1 / 2}^{n-1}$ involve only know quantities if we assume that solution is known on $y=y^{n-1}$. To linearize the nonlinear system of equations (A26) to (A32) using Newton's method, we introduce the following iterates:

$$
\begin{array}{lll}
F_{j}^{(i+1)}=F_{j}^{(i)}+\delta F_{j}^{(i)}, & u_{j}^{(i+1)}=u_{j}^{(i)}+\delta u_{j}^{(i)}, & v_{j}^{(i+1)}=v_{j}^{(i)}+\delta v_{j}^{(i)}, \\
G_{j}^{(i+1)}=G_{j}^{(i)}+\delta G_{j}^{(i)}, & t_{j}^{(i+1)}=t_{j}^{(i)}+\delta t_{j}^{(i)}, \\
H_{j}^{(i+1)}=H_{j}^{(i)}+\delta H_{j}^{(i)}, & p_{j}^{(i+1)}=p_{j}^{(i)}+\delta p_{j}^{(i)} .
\end{array}
$$

Then we substitute these expressions into equations (A26) - (A32) except for the term, $y^{n-1}$, and this yields:

$$
\begin{aligned}
& \left(F_{j}^{(i)}+\delta F_{j}^{(i)}\right)-\left(F_{j-1}^{(i)}+\delta F_{j-1}^{(i)}\right)-\frac{h_{j}}{2}\left(u_{j}^{(i)}+\delta u_{j}^{(i)}+u_{j-1}^{(i)}+\delta u_{j-1}^{(i)}\right)=0, \\
& \left(u_{j}^{(i)}+\delta u_{j}^{(i)}\right)-\left(u_{j-1}^{(i)}+\delta u_{j-1}^{(i)}\right)-\frac{h_{j}}{2}\left(v_{j}^{(i)}+\delta v_{j}^{(i)}+v_{j-1}^{(i)}+\delta v_{j-1}^{(i)}\right)=0, \\
& \left(G_{j}^{(i)}+\delta G_{j}^{(i)}\right)-\left(G_{j-1}^{(i)}+\delta g_{j-1}^{(i)}\right)-\frac{h_{j}}{2}\left(t_{j}^{(i)}+\delta t_{j}^{(i)}+t_{j-1}^{(i)}+\delta t_{j-1}^{(i)}\right)=0, \\
& \left(H_{j}^{(i)}+\delta H_{j}^{(i)}\right)-\left(H_{j-1}^{(i)}+\delta H_{j-1}^{(i)}\right)-\frac{h_{j}}{2}\left(p_{j}^{(i)}+\delta p_{j}^{(i)}+p_{j-1}^{(i)}+\delta p_{j-1}^{(i)}\right)=0, \\
& {\left[\left(v_{j}^{(i)}+\delta v_{j}^{(i)}\right)-\left(v_{j-1}^{(i)}+\delta v_{j-1}^{(i)}\right)\right]+\frac{h_{j}}{2}\left[\left(F_{j}^{(i)}+\delta F_{j}^{(i)}+F_{j-1}^{(i)}+\delta F_{j-1}^{(i)}\right)\left(v_{j}^{(i)}+\delta v_{j}^{(i)}+v_{j-1}^{(i)}+\delta v_{j-1}^{(i)}\right)\right]} \\
& \quad-\frac{h_{j}}{4}\left(u_{j}^{(i)}+\delta u_{j}^{(i)}+u_{j-1}^{(i)}+\delta u_{j-1}^{(i)}\right)^{2}-\frac{h_{j}}{2}\left(\frac{1}{\kappa_{x}}+N m\right)\left(u_{j}^{(i)}+\delta u_{j}^{(i)}+u_{j-1}^{(i)}+\delta u_{j-1}^{(i)}\right) \\
& \quad+\frac{\varepsilon h_{j}}{4}\left(G_{j}^{(i)}+\delta G_{j}^{(i)}+G_{j-1}^{(i)}+\delta G_{j-1}^{(i)}\right)^{2}+\frac{h_{j}}{2}\left(H_{j}^{(i)}+\delta H_{j}^{(i)}+H_{j-1}^{(i)}+\delta H_{j-1}^{(i)}\right)=\left[R_{1}\right]_{j-1 / 2}^{n-1}, \\
& \quad-\left(\frac{1}{\kappa_{\theta}}+N m\right) \frac{h_{j}}{2}\left(G_{j}^{(i)}+\delta G_{j}^{(i)}+G_{j-1}^{(i)}+\delta G_{j-1}^{(i)}\right)=\left[R_{2}\right]_{j-1 / 2}^{n-1}, \\
& {\left[\left(t_{j}^{(i)}+\delta t_{j}^{(i)}\right)-\left(t_{j-1}^{(i)}+\delta t_{j-1}^{(i)}\right)\right]+\frac{h_{j}}{2}\left[\left(F_{j}^{(i)}+\delta F_{j}^{(i)}+F_{j-1}^{(i)}+\delta F_{j-1}^{(i)}\right)\left(t_{j}^{(i)}+\delta t_{j}^{(i)}+t_{j-1}^{(i)}+\delta t_{j-1}^{(i)}\right)\right]} \\
& \left.\left.\quad u_{j}^{(i)}+u_{j-1}^{(i)}+\delta u_{j-1}^{(i)}\right)\left(G_{j}^{(i)}+\delta G_{j}^{(i)}+G_{j-1}^{(i)}+\delta G_{j-1}^{(i)}\right)\right]
\end{aligned}
$$




$$
\begin{aligned}
\frac{1}{\operatorname{Pr}} & {\left[\left(p_{j}^{(i)}+\delta p_{j}^{(i)}\right)-\left(p_{j-1}^{(i)}+\delta p_{j-1}^{(i)}\right)\right]+\frac{h_{j}}{2}\left[\left(F_{j}^{(i)}+\delta F_{j}^{(i)}+F_{j-1}^{(i)}+\delta F_{j-1}^{(i)}\right)\left(p_{j}^{(i)}+\delta p_{j}^{(i)}+p_{j-1}^{(i)}+\delta p_{j-1}^{(i)}\right)\right] } \\
& -\frac{h_{j}}{4}\left[\left(u_{j}^{(i)}+\delta u_{j}^{(i)}+u_{j-1}^{(i)}+\delta u_{j-1}^{(i)}\right)\left(H_{j}^{(i)}+\delta H_{j}^{(i)}+H_{j-1}^{(i)}+\delta H_{j-1}^{(i)}\right)\right]=\left[R_{3}\right]_{j-1 / 2}^{n-1} .
\end{aligned}
$$

Next we drop the terms that are quadratic in the following $\left(\delta F_{j}^{(i)}, \delta u_{j}^{(i)}, \delta v_{j}^{(i)}, \delta G_{j}^{(i)}, \delta t_{j}^{(i)}, \delta H_{j}^{(i)}, \delta p_{j}^{(i)}\right)$. We also drop the superscript $I$ for simplicity. After some algebraic manipulations, the following linear tridiagonal system of equations is obtained:

$$
\begin{aligned}
& \delta F_{j}-\delta F_{j-1}-\frac{h_{j}}{2}\left(\delta u_{j}+\delta u_{j-1}\right)=\left(r_{1}\right)_{j-1 / 2} \\
& \delta u_{j}-\delta u_{j-1}-\frac{h_{j}}{2}\left(\delta v_{j}+\delta v_{j-1}\right)=\left(r_{2}\right)_{j-1 / 2} \\
& \delta G_{j}-\delta G_{j-1}-\frac{h_{j}}{2}\left(\delta t_{j}+\delta t_{j-1}\right)=\left(r_{3}\right)_{j-1 / 2} \\
& \delta H_{j}-\delta H_{j-1}-\frac{h_{j}}{2}\left(\delta t_{j}+\delta t_{j-1}\right)=\left(r_{4}\right)_{j-1 / 2} \\
& \left(a_{1}\right)_{j} \delta v_{j}+\left(a_{2}\right)_{j} \delta v_{j-1}+\left(a_{3}\right)_{j} \delta F_{j}+\left(a_{4}\right)_{j} \delta F_{j-1}+\left(a_{5}\right)_{j} \delta u_{j}+\left(a_{6}\right)_{j} \delta u_{j-1} \\
& +\left(a_{7}\right)_{j} \delta G_{j}+\left(a_{8}\right)_{j} \delta G_{j-1}+\left(a_{9}\right)_{j} \delta H_{j}+\left(a_{10}\right)_{j} \delta H_{j-1}=\left(r_{5}\right)_{j-1 / 2}, \\
& \left(b_{1}\right)_{j} \delta t_{j}+\left(b_{2}\right)_{j} \delta t_{j-1}+\left(b_{3}\right)_{j} \delta F_{j}+\left(b_{4}\right)_{j} \delta F_{j-1}+\left(b_{5}\right)_{j} \delta u_{j}+\left(b_{6}\right)_{j} \delta u_{j-1} \\
& +\left(b_{7}\right)_{j} \delta G_{j}+\left(b_{8}\right)_{j} \delta G_{j-1}=\left(r_{6}\right)_{j-1 / 2}, \\
& \left(c_{1}\right)_{j} \delta p_{j}+\left(c_{2}\right)_{j} \delta p_{j-1}+\left(c_{3}\right)_{j} \delta F_{j}+\left(c_{4}\right)_{j} \delta F_{j-1}+\left(c_{5}\right)_{j} \delta u_{j}+\left(c_{6}\right)_{j} \delta u_{j-1} \\
& +\left(c_{7}\right)_{j} \delta H_{j}+\left(c_{8}\right)_{j} \delta H_{j-1}=\left(r_{7}\right)_{j-1 / 2},
\end{aligned}
$$

where:

$$
\begin{array}{ll}
\left(a_{1}\right)_{j}=1+h_{j} F_{j-1 / 2}, & \left(a_{2}\right)_{j}=-1+h_{j} F_{j-1 / 2}, \\
\left(a_{3}\right)_{j}=h_{j} v_{j-1 / 2}, & \left(a_{4}\right)_{j}=\left(a_{3}\right)_{j},
\end{array}
$$




$$
\begin{aligned}
& \left(a_{5}\right)_{j}=h_{j}\left[u_{j-1 / 2}-\frac{1}{2}\left(\frac{1}{\kappa_{x}}+N m\right)\right], \quad\left(a_{8}\right)_{j}=\left(a_{7}\right)_{j}, \\
& \left(a_{7}\right)_{j}=\varepsilon h_{j} G_{j-1 / 2}, \\
& \left(a_{9}\right)_{j}=\frac{1}{2} h_{j}, \quad \quad\left(a_{10}\right)_{j}=\left(a_{9}\right)_{j}, \\
& \left(b_{1}\right)_{j}=1+h_{j} F_{j-1 / 2}, \quad\left(b_{2}\right)_{j}=-1+h_{j} F_{j-1 / 2}, \\
& \left(b_{3}\right)_{j}=h_{j} t_{j-1 / 2}, \quad\left(b_{4}\right)_{j}=\left(b_{3}\right)_{j}, \\
& \left(b_{5}\right)_{j}=-h_{j} G_{j-1 / 2}, \quad\left(b_{6}\right)_{j}=\left(b_{5}\right)_{j}, \\
& \left(b_{7}\right)_{j}=h_{j}\left[-u_{j-1 / 2}-\frac{1}{2}\left(\frac{1}{\kappa_{\theta}}+N m\right), \quad\left(b_{8}\right)_{j}=\left(b_{7}\right)_{j},\right.
\end{aligned}
$$

$$
\left(c_{1}\right)_{j}=\frac{1}{\operatorname{Pr}}+h_{j} F_{j-1 / 2}, \quad\left(c_{2}\right)_{j}=-\frac{1}{\operatorname{Pr}}+h_{j} F_{j-1 / 2},
$$$$
\left(c_{3}\right)_{j}=h_{j} p_{j-1 / 2}, \quad\left(c_{4}\right)_{j}=\left(c_{3}\right)_{j} \text {, }
$$

$$
\begin{array}{ll}
\left(c_{5}\right)_{j}=-\frac{h_{j}}{2} H_{j-1 / 2}, & \left(c_{6}\right)_{j}=\left(c_{5}\right)_{j}, \\
\left(c_{7}\right)_{j}=-\frac{h_{j}}{2} u_{j-1 / 2}, & \left(c_{8}\right)_{j}=\left(c_{7}\right)_{j},
\end{array}
$$

$$
\begin{array}{ll}
\left(r_{1}\right)_{j-1 / 2}=F_{j-1}-F_{j}+h_{j} u_{j-1 / 2}, & \left(r_{2}\right)_{j-1 / 2}=u_{j-1}-u_{j}+h_{j} v_{j-1 / 2}, \\
\left(r_{3}\right)_{j-1 / 2}=G_{j-1}-G_{j}+h_{j} t_{j-1 / 2}, & \left(r_{4}\right)_{j-1 / 2}=H_{j-1}-H_{j}+h_{j} p_{j-1 / 2},
\end{array}
$$$$
\left(r_{5}\right)_{j-1 / 2}=\left(v_{j-1}-v_{j}\right)-2 h_{j} F_{j-1 / 2} v_{j-1 / 2}+h_{j} u_{j-1 / 2}^{2}+\left(\frac{1}{\kappa_{x}}+N m\right) h_{j} u_{j-1 / 2}
$$$$
-\varepsilon h_{j} G_{j-1 / 2}^{2}-h_{j} H_{j-1 / 2}+\left[R_{1}\right]_{j-1 / 2}^{n-1}
$$

$\left(r_{6}\right)_{j-1 / 2}=\left(t_{j-1}-t_{j}\right)-2 h_{j} F_{j-1 / 2} t_{j-1 / 2}+2 h_{j} u_{j-1 / 2} G_{j-1 / 2}+h_{j}\left(\frac{1}{\kappa_{\theta}}+N m\right) G_{j-1 / 2}+\left[R_{2}\right]_{j-1 / 2}^{n-1}$,

$\left(r_{7}\right)_{j-1 / 2}=\frac{1}{\operatorname{Pr}}\left(p_{j-1}-p_{j}\right)-2 h_{j} F_{j-1 / 2} p_{j-1 / 2}+h_{j} u_{j-1 / 2} H_{j-1 / 2}+\left[R_{3}\right]_{j-1 / 2}^{n-1}$. 
To complete the system (A44) to (A50) we recall the boundary conditions (A24), which can be satisfied exactly with no iteration. Therefore to maintain these correct values in all the iterates, we take:

$$
\delta F_{0}=0, \quad \delta u_{0}=0, \quad \delta G_{0}=0, \quad \delta H_{0}=0, \quad \delta u_{J}=0, \quad \delta G_{J}=0, \quad \delta H_{J}=0
$$

\section{Phase d) Block-tridiagonal Elimination of Linear Keller Algebraic Equations}

The linear system (A44) to (A50) can now be solved by the block-elimination method.

The linearized difference equations of this system have a block-tridiagonal structure. Commonly, the block-tridiagonal structure consists of variables or constants, however here, an interesting feature can be observed that is, for the Keller-box method, it consists of block matrices. Intrinsic to the block-elimination method used in the Keller-box implicit finite difference method, is the correct derivation of the elements of the block matrices from the linear system. We consider three cases, namely when $j=1, J-1$ and $J$. When $j=1$, the linear system equations become:

$$
\begin{aligned}
& \delta F_{1}-\delta F_{0}-\frac{h_{1}}{2}\left(\delta u_{1}+\delta u_{0}\right)=\left(r_{1}\right)_{1-1 / 2}, \\
& \delta u_{1}-\delta u_{0}-\frac{h_{j}}{2}\left(\delta v_{1}+\delta v_{0}\right)=\left(r_{2}\right)_{1-1 / 2} \\
& \delta G_{1}-\delta G_{0}-\frac{h_{j}}{2}\left(\delta t_{1}+\delta t_{0}\right)=\left(r_{3}\right)_{1-1 / 2} \\
& \delta H_{1}-\delta H_{0}-\frac{h_{j}}{2}\left(\delta p_{1}+\delta p_{0}\right)=\left(r_{4}\right)_{1-1 / 2} \\
& \left(a_{1}\right)_{1} \delta v_{1}+\left(a_{2}\right)_{1} \delta v_{0}+\left(a_{3}\right)_{1} \delta F_{1}+\left(a_{4}\right)_{1} \delta F_{0}+\left(a_{5}\right)_{1} \delta u_{1}+\left(a_{6}\right)_{1} \delta u_{0} \\
& +\left(a_{7}\right)_{1} \delta G_{1}+\left(a_{8}\right)_{1} \delta G_{0}+\left(a_{9}\right)_{1} \delta H_{1}+\left(a_{10}\right)_{1} \delta H_{0}=\left(r_{5}\right)_{1-1 / 2}, \\
& \left(b_{1}\right)_{1} \delta t_{1}+\left(b_{2}\right)_{1} \delta t_{0}+\left(b_{3}\right)_{1} \delta F_{1}+\left(b_{4}\right)_{1} \delta F_{0}+\left(b_{5}\right)_{1} \delta u_{1}+\left(b_{6}\right)_{1} \delta u_{0} \\
& +\left(b_{7}\right)_{1} \delta G_{1}+\left(b_{8}\right)_{1} \delta G_{0}=\left(r_{6}\right)_{1-1 / 2} \text {, } \\
& \left(c_{1}\right)_{1} \delta p_{1}+\left(c_{2}\right)_{1} \delta p_{0}+\left(c_{3}\right)_{1} \delta F_{1}+\left(c_{4}\right)_{1} \delta F_{0}+\left(c_{5}\right)_{1} \delta u_{1}+\left(c_{6}\right)_{1} \delta u_{0} \\
& +\left(c_{7}\right)_{1} \delta H_{1}+\left(c_{8}\right)_{1} \delta H_{0}=\left(r_{7}\right)_{1-1 / 2} \text {, }
\end{aligned}
$$

Designating $d_{1}=-\frac{1}{2} h_{1}$, and $\delta F_{0}=0, \delta u_{0}=0, \delta G_{0}=0, \delta H_{0}=0 \quad$ the corresponding matrix form assumes: 


$$
\begin{gathered}
{\left[\begin{array}{ccccccc}
0 & 0 & 0 & 1 & 0 & 0 & 0 \\
d_{1} & 0 & 0 & 0 & d_{1} & 0 & 0 \\
0 & d_{1} & 0 & 0 & 0 & d_{1} & 0 \\
0 & 0 & d_{1} & 0 & 0 & 0 & d_{1} \\
\left(a_{2}\right)_{1} & 0 & 0 & \left(a_{3}\right)_{1} & \left(a_{1}\right)_{1} & 0 & 0 \\
0 & \left(b_{2}\right)_{1} & 0 & \left(b_{3}\right)_{1} & 0 & \left(b_{1}\right)_{1} & 0 \\
0 & 0 & \left(c_{2}\right)_{1} & \left(c_{3}\right)_{1} & 0 & 0 & \left(c_{1}\right)_{1}
\end{array}\right]\left[\begin{array}{l}
\delta v_{0} \\
\delta t_{0} \\
\delta p_{0} \\
\delta F_{1} \\
\delta v_{1} \\
\delta t_{1} \\
\delta p_{1}
\end{array}\right]+} \\
{\left[\begin{array}{ccccccc}
d_{1} & 0 & 0 & 0 & 0 & 0 & 0 \\
1 & 0 & 0 & 0 & 0 & 0 & 0 \\
0 & 1 & 0 & 0 & 0 & 0 & 0 \\
0 & 0 & 1 & 0 & 0 & 0 & 0 \\
\left(a_{5}\right)_{1} & \left(a_{7}\right)_{1} & \left(a_{9}\right)_{1} & 0 & 0 & 0 & 0 \\
\left(b_{5}\right)_{1} & \left(b_{7}\right)_{1} & 0 & 0 & 0 & 0 & 0 \\
\left(c_{5}\right)_{1} & 0 & \left(c_{7}\right)_{1} & 0 & 0 & 0 & 0
\end{array}\right]\left[\begin{array}{l}
\delta_{1} \\
\delta G_{1} \\
\delta H_{1} \\
\delta F_{2} \\
\delta v_{2} \\
\delta t_{2} \\
\delta p_{2}
\end{array}\right]=\left[\begin{array}{l}
\left(r_{1}\right)_{1-(1 / 2)} \\
\left(r_{2}\right)_{1-(1 / 2)} \\
\left(r_{3}\right)_{1-(1 / 2)} \\
\left(r_{4}\right)_{1-(1 / 2)} \\
\left(r_{5}\right)_{1-(1 / 2)} \\
\left(r_{6}\right)_{1-(1 / 2)} \\
\left(r_{7}\right)_{1-(1 / 2)}
\end{array}\right]}
\end{gathered}
$$

For $j=1$, we have $\left[A_{1}\right]\left[\delta_{1}\right]+\left[C_{1}\right]\left[\delta_{2}\right]=\left[r_{1}\right]$. Similar procedures are followed at the different stations. Effectively the seven linearized finite difference equations have the matrix-vector form:

$$
\Lambda \delta_{\mathrm{j}}=\zeta_{\mathrm{j}}
$$

where $\Lambda=$ Keller coefficient matrix of order $7 \times 7, \delta_{\mathbf{j}}=7^{\mathrm{TH}}$ order vector for errors (perturbation) quantities and $\zeta_{\mathbf{j}}=$ seventh order vector for Keller residuals. This system is then recast as an expanded matrix-vector system, viz:

$$
\varsigma_{j} \delta_{j}-\omega_{j} \delta_{j}=\zeta_{j}
$$

where now $\varsigma_{\mathbf{j}}=$ coefficient matrix of order $7 \times 7, \omega_{\mathbf{j}}=$ coefficient matrix of order $7 \times 7$ and $\zeta_{\mathrm{j}}=$ seventh order vector of errors (iterates) at previous station on grid. Finally the 
complete linearized system is formulated as a block matrix system where each element in the coefficient matrix is a matrix itself. 


\section{FIGURES}

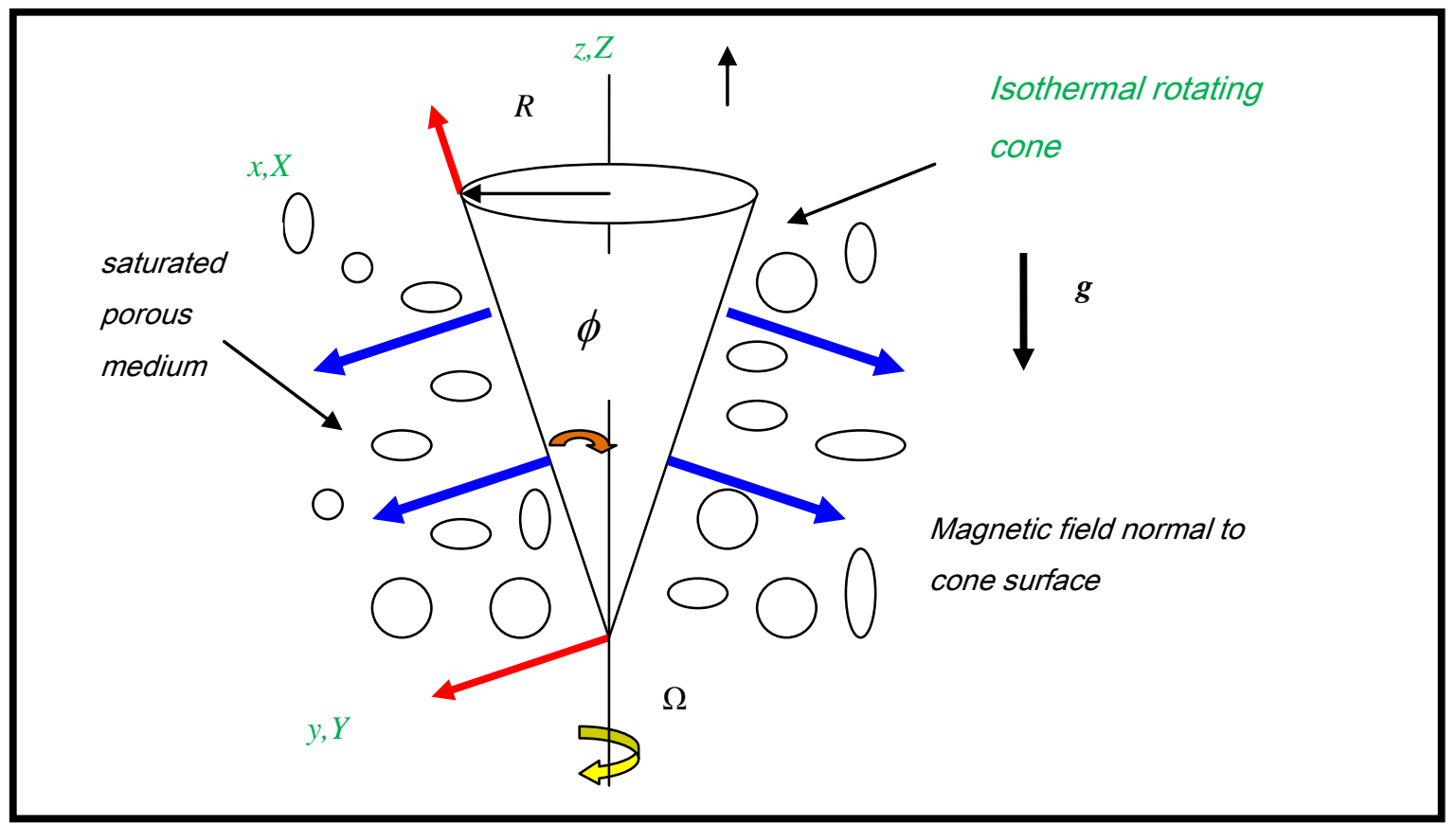

Figure 1: Physical model for magneto-convection from a rotating cone in an orthotropic porous medium

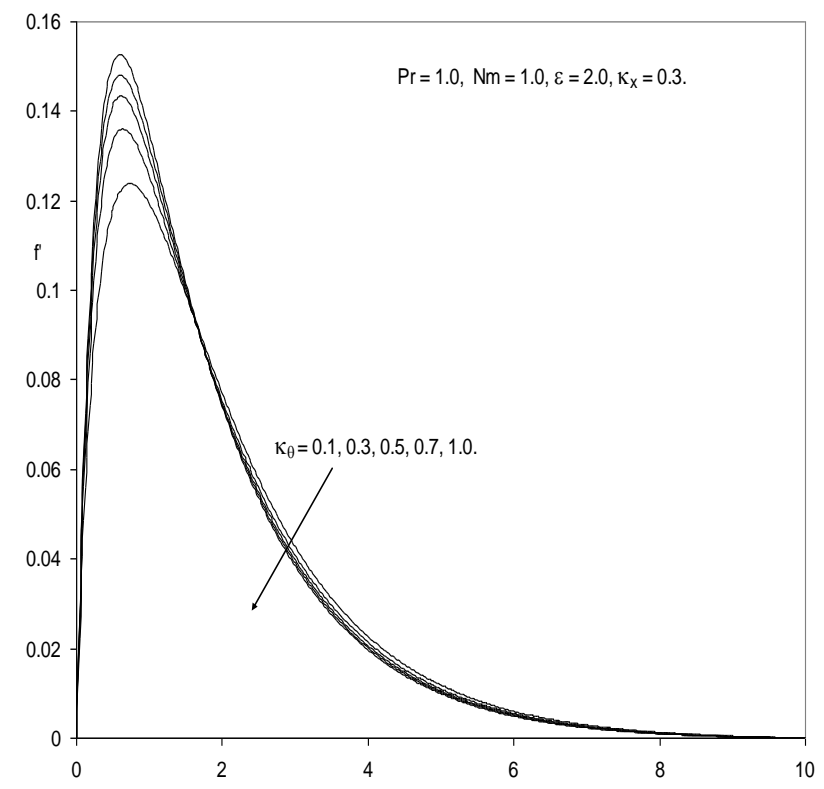

Figure 2: Tangential velocity distribution for various $\theta$-direction Darcy numbers 


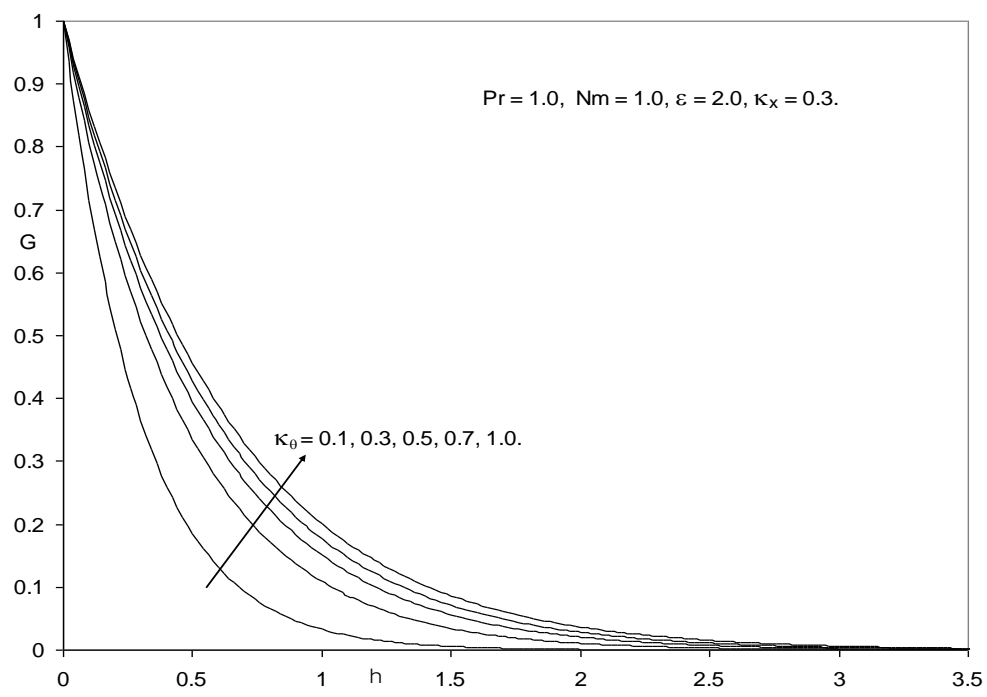

Figure 3: Swirl velocity distribution for various $\theta$-direction Darcy numbers

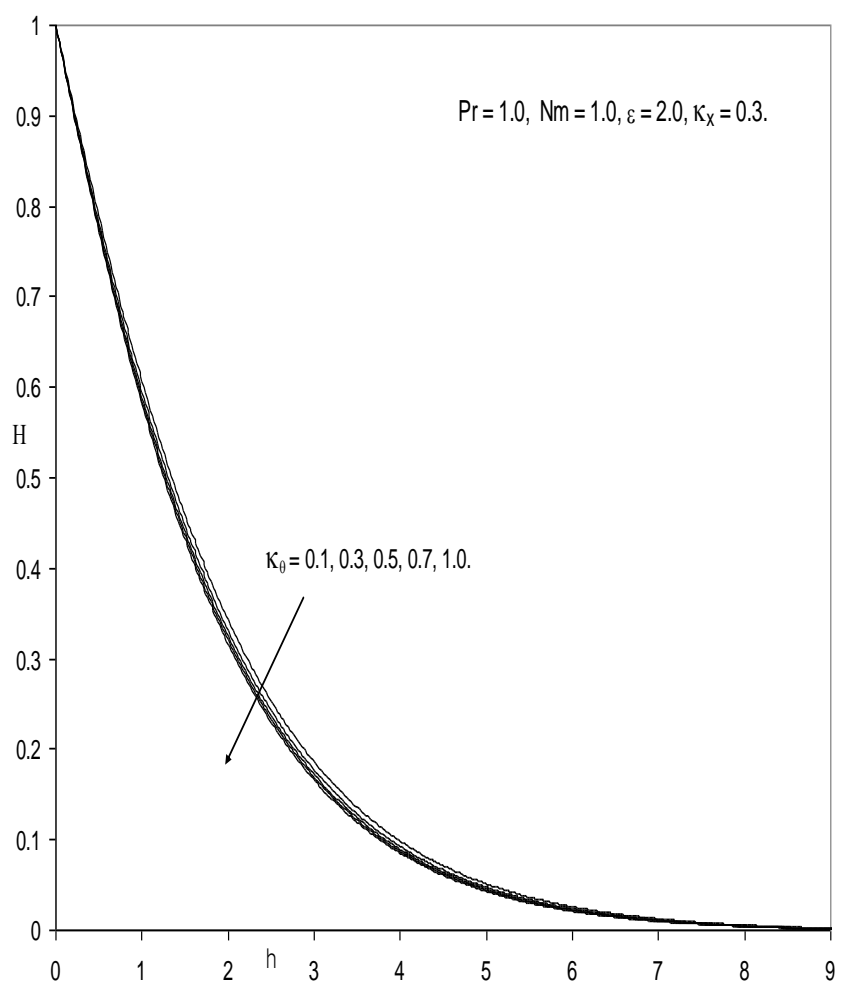

Figure 4: Temperature distribution for various $\theta$-direction Darcy numbers 


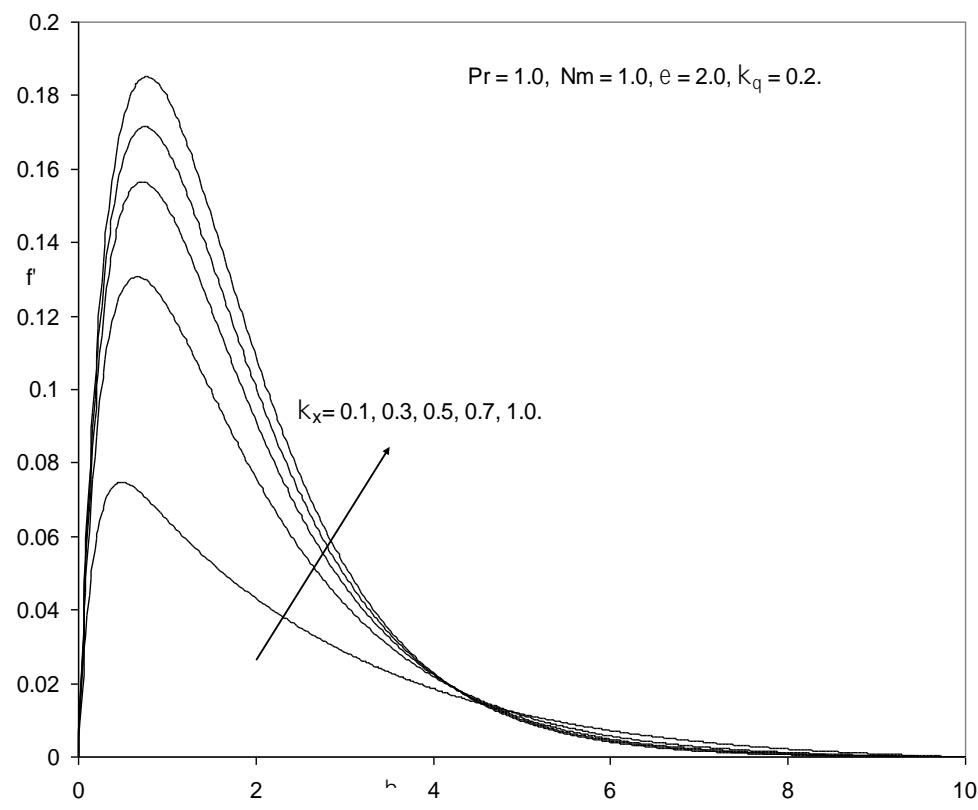

Figure 5: Tangential velocity distribution for various $x$-direction Darcy numbers

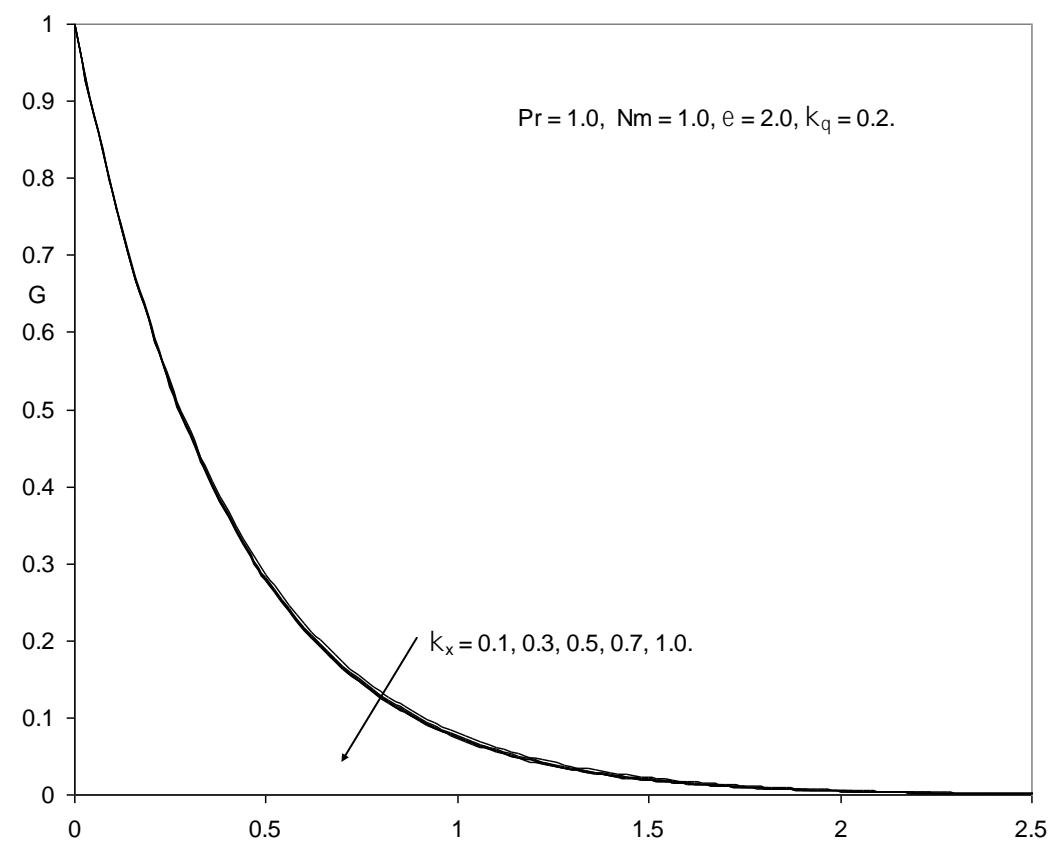

Figure 6: Swirl velocity distribution for various $x$-direction Darcy numbers 


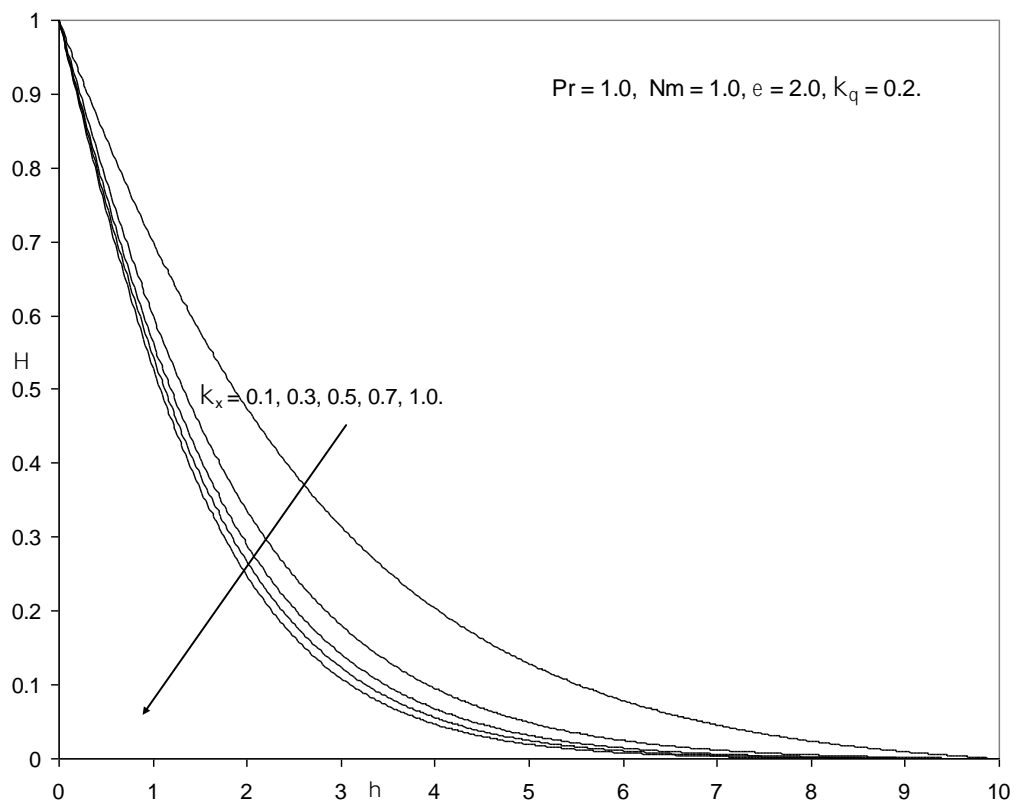

Figure 7: Temperature distribution for various $x$-direction Darcy numbers

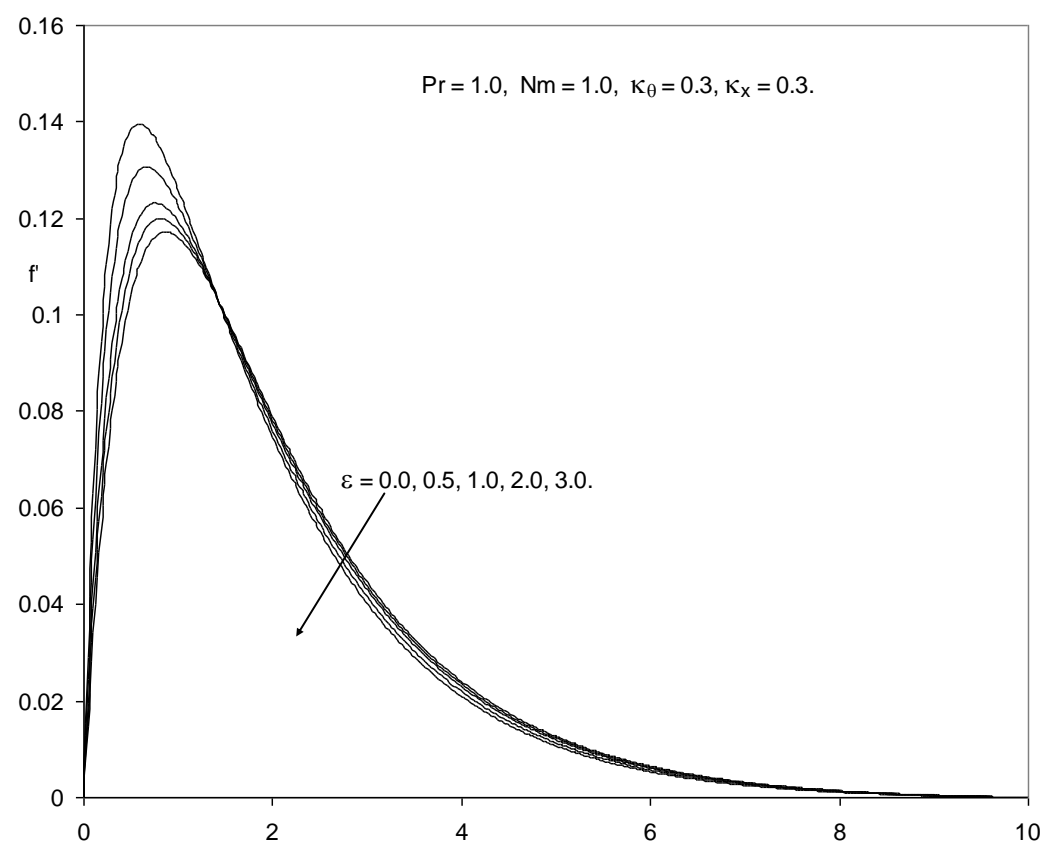

Figure 8: Tangential velocity distribution for various spin parameters 


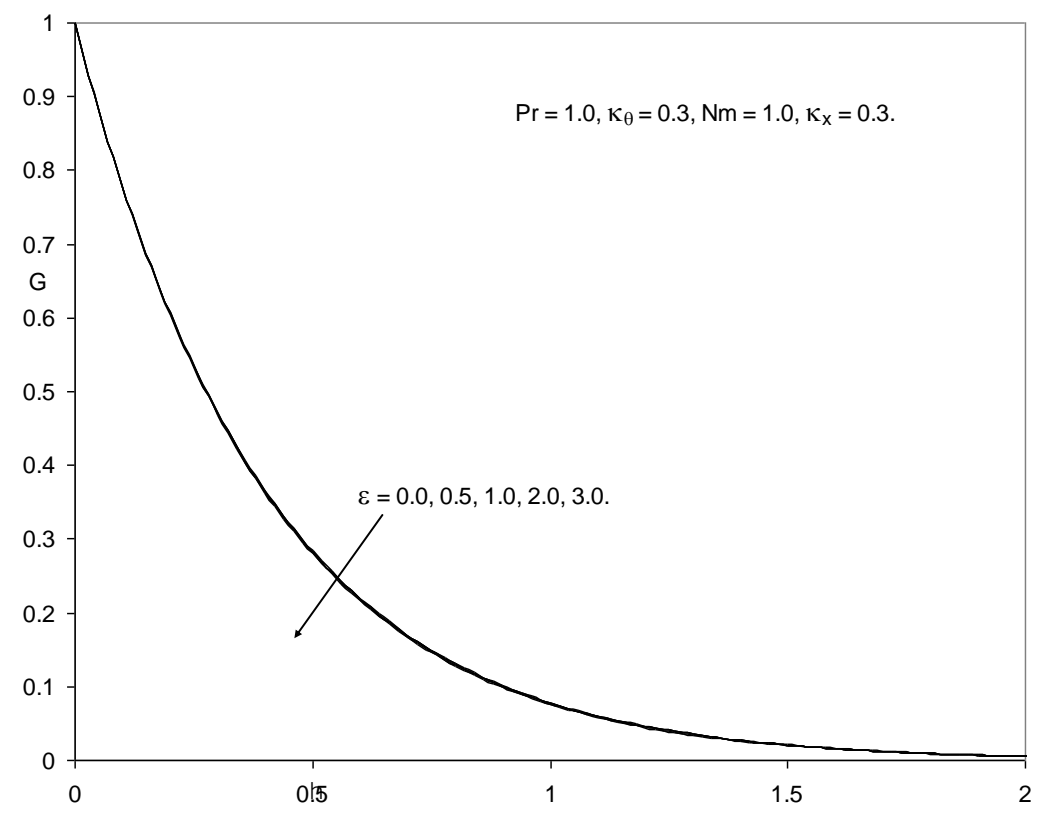

Figure 9: Swirl velocity distribution for various spin parameters

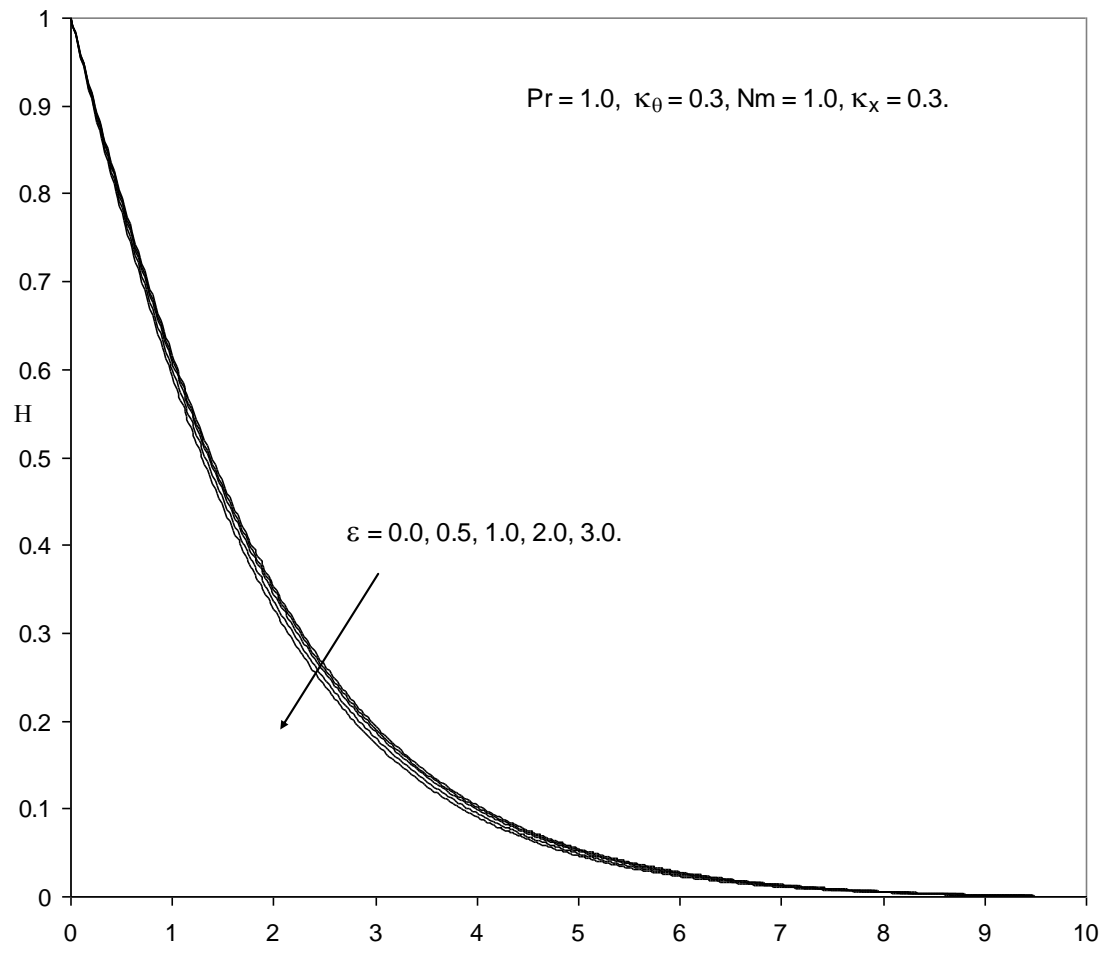

Figure 10: Temperature distribution for various spin parameters 


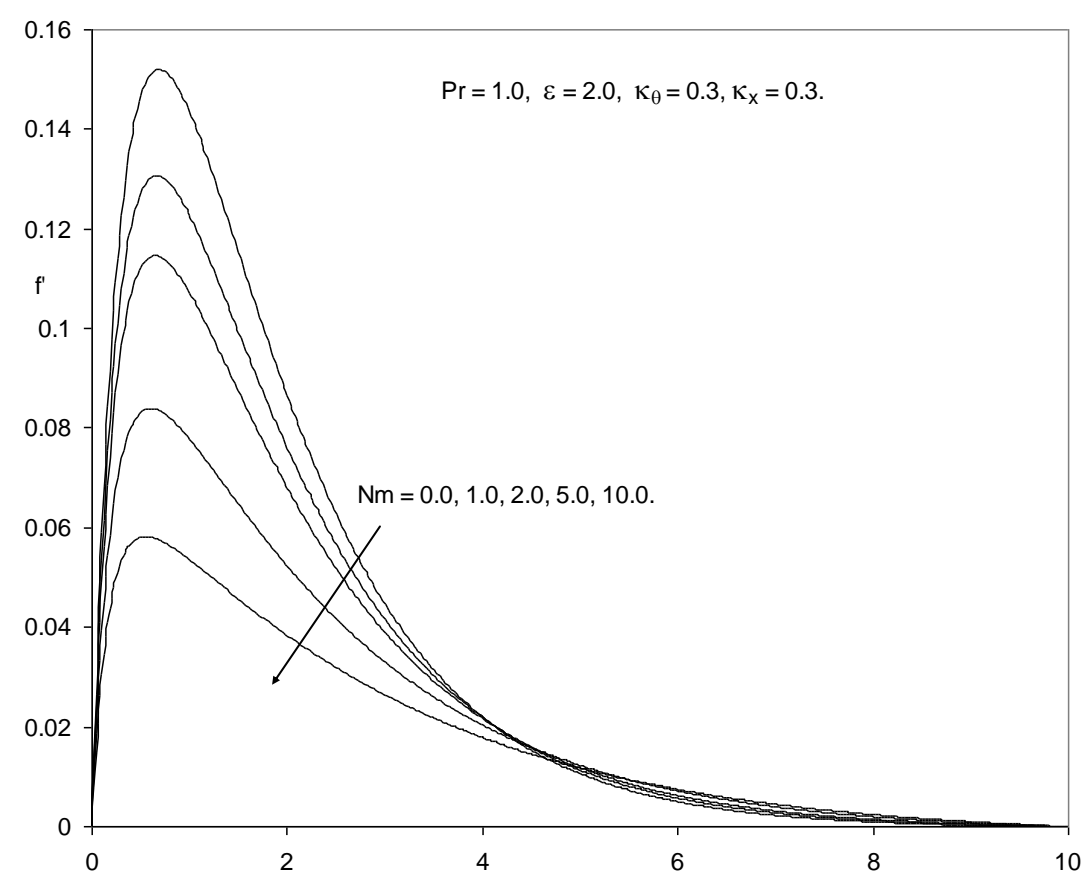

Figure 11: Tangential velocity distribution for various magnetic parameters

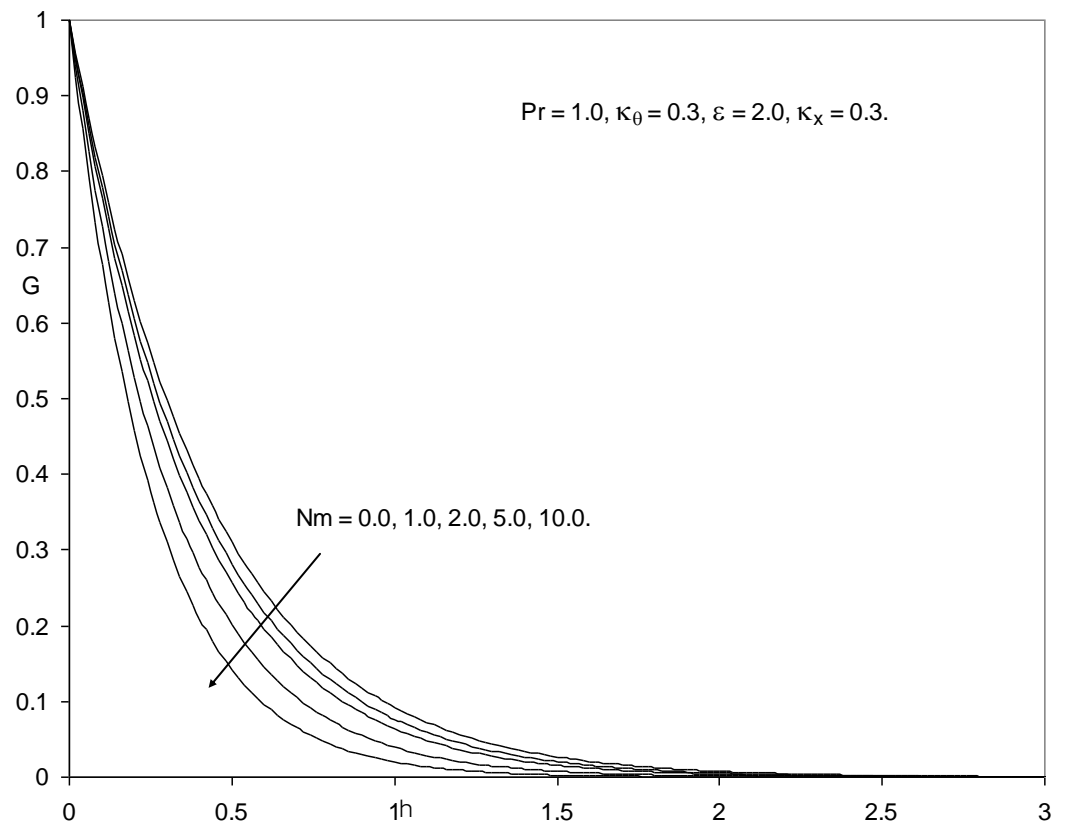

Figure 12: Swirl velocity distribution for various magnetic parameters 


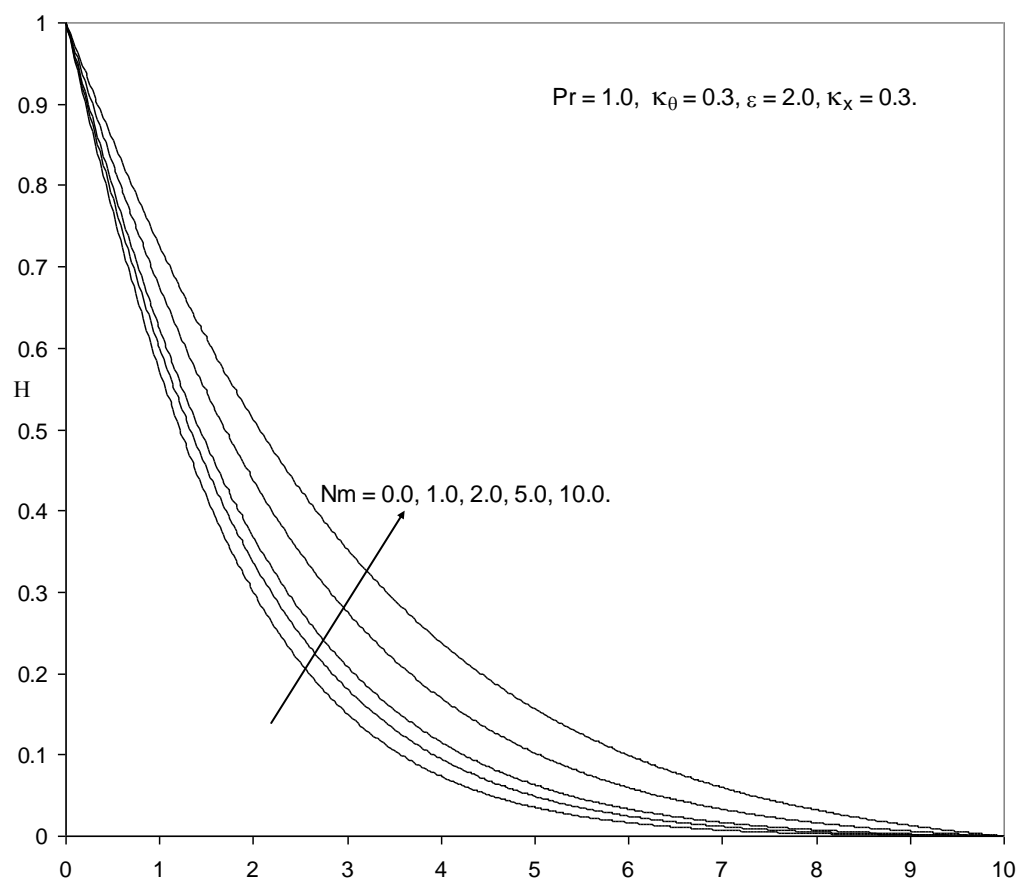

Figure 13: Temperature distribution for various magnetic parameters

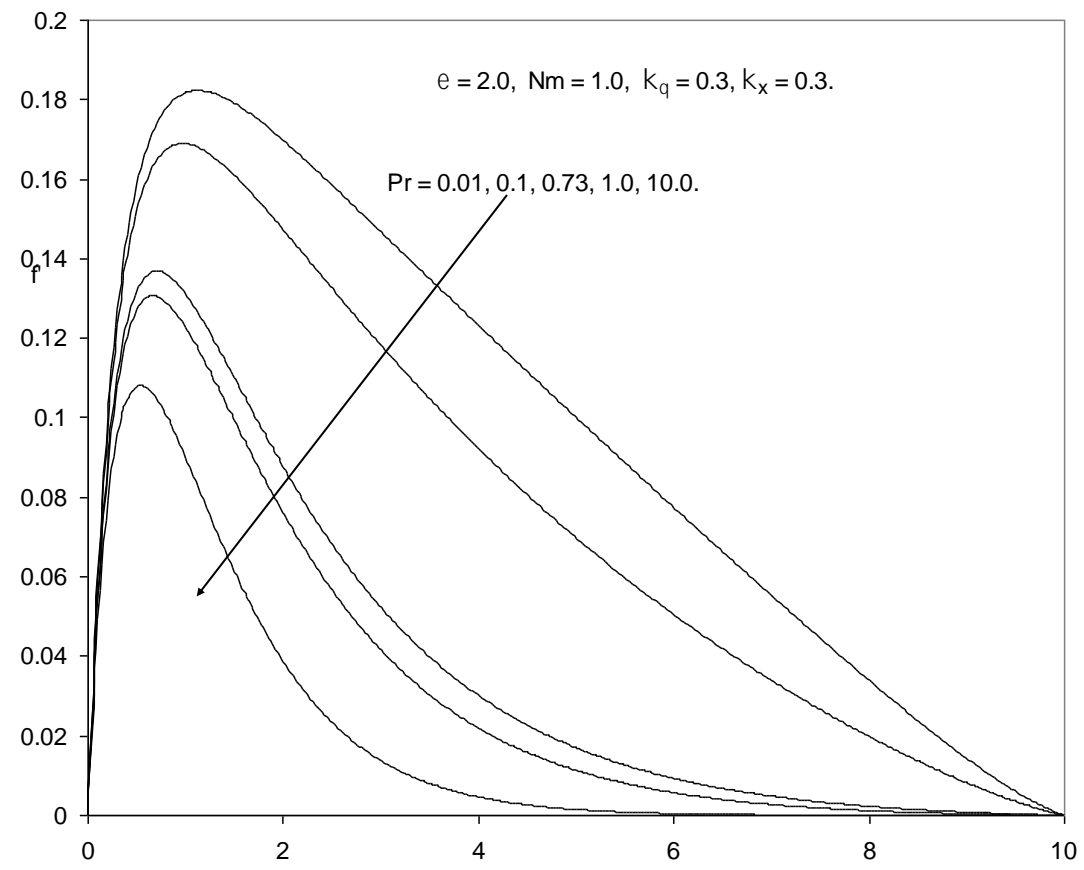

Figure 14: Tangential velocity distribution for various Prandtl numbers 


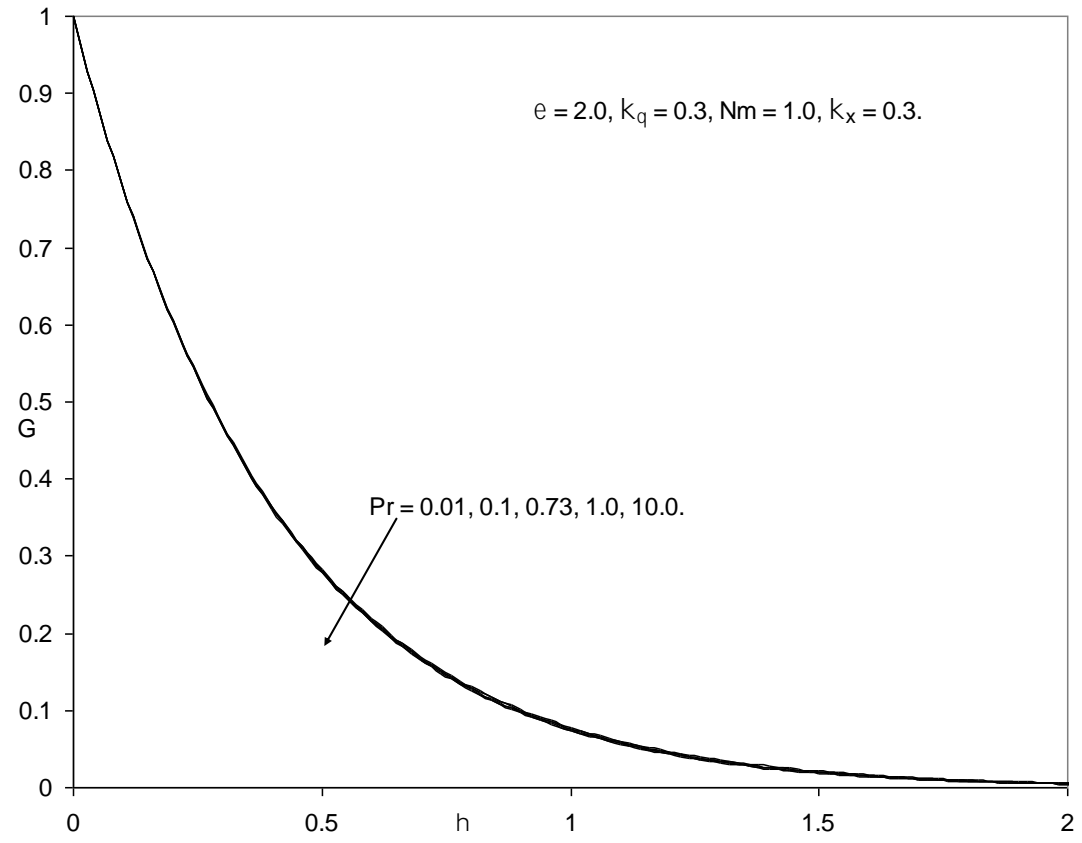

Figure 15: Swirl velocity distribution for various Prandtl numbers

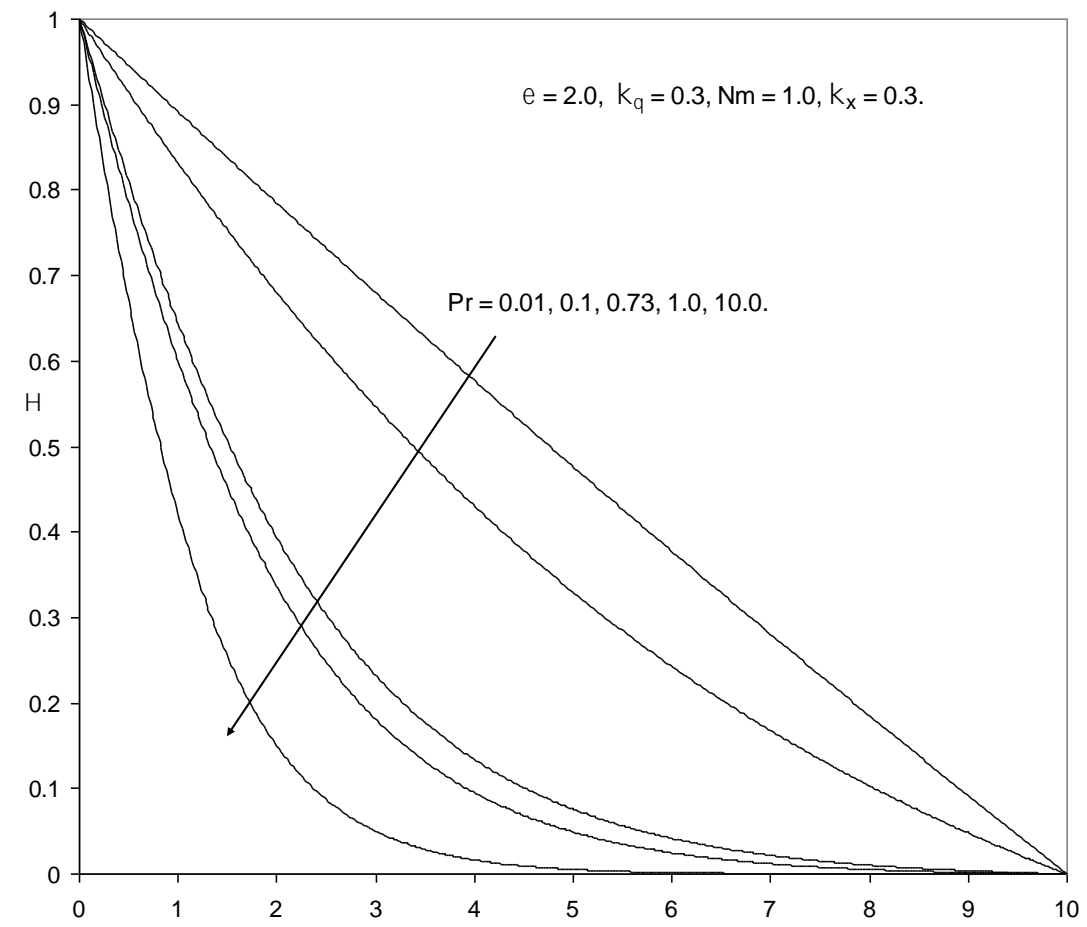

Figure 16: Temperature distribution for various Prandtl numbers 